\title{
Pairwise Soft Connected in Soft Bitopological Spaces
}

\author{
Ali Kandil \\ Department of Mathematics, \\ Faculty of Science, \\ Helwan University, \\ Helwan, Egypt
}

\author{
Osama El-Tantawy \\ Department of Mathematics, \\ Faculty of Science, \\ Zagazig University, \\ Zagazig, Egypt \\ Shawqi Ahmed Hazza \\ Department of Mathematics, \\ Faculty of Education, \\ Taiz University, \\ Taiz, Yemen
}

\author{
Sobhy El-Sheikh \\ Department of Mathematics, \\ Faculty of Education, \\ Ain Shams University, \\ Cairo, Egypt
}

\begin{abstract}
In this paper, we introduce the notion of $p$-separated soft sets based on the soft space $\left(X, \eta_{12}, E\right)$ which generate by soft bitopological space $\left(X, \eta_{1}, \eta_{2}, E\right)$ and study some of its properties. Based on this notion we introduce the notions of $p$ -soft connected(disconnected) spaces and study some of their characterizations and properties. Also, we study the connected of $p$-soft sets by using the soft space $\left(X, \eta_{12}, E\right)$. Some examples have given to support these concepts.
\end{abstract}

\section{Keywords}

Soft set; Soft topology; Soft bitopological spaces; $p$-separated sets; $p$-separated soft sets; $p$-soft connected spaces; $p$-soft disconnected spaces; $p$-connected soft set and pairwise soft continuous.

\section{INTRODUCTION}

Molodtsov [19] initiated a novel concept of soft set theory, which is a completely new approach for modeling vagueness and uncertainty. He successfully applied the soft set theory to several directions such as smoothness of functions, game theory, Riemann Integration, and theory of measurement. In recent years, development in the fields of soft set theory and its application has been taking place in a rapid pace. This is because of the general nature of parameterized expressed by a soft set. Shabir and Naz [26] introduced the notion of soft topological spaces which are defined over an initial universe with a fixed set of parameters. I. Zorlutuna et al. [28] studied some new properties of soft continuous mappings and gave some new characterizations of sot continuous, soft open, soft closed mappings and also soft homeomorphisms. In 2012, E. Peyghan, B. Samadi and A. Tayebi [?] introduced and explore the notions of soft connectedness in soft topological spaces. Also, in 2014, M. Al-Khafaj and M. Mahmood [1] introduced and studied some properties of soft connected spaces and soft locally connected spaces. Ittanagi [10] introduced the concept of soft bitopological space and studied some types of soft separation axiom for soft bitopological spaces from his point of view. Kandil et al. [[13],[16]]introduced some structures of soft bitopological spaces. Recently, Kandil et al. [15] introduced some types of pairwise soft open (continuous) mappings and some related results.

The purpose of this article is to introduce and study the concept of soft connectedness in soft bitopological spaces. We study the concepts of pairwise separated soft sets, pairwise soft connected (disconnected) spaces and pairwise connected soft sets. The rest of this paper is organized as follows. In section (2) we introduced briefly the notions of soft set, soft topology, soft bitopological spaces, soft mapping and some related topics. In Section (3), we introduce the notion of pairwise separated soft sets and give some characterizations of these soft sets. In Section (3), we introduce the notions of pairwise soft connected (disconnected) spaces and investigate some of their properties. In section (5), we give the concept of $p$-connected soft sets and some related properties are studied. The last section summarizes the conclusions.

\section{2. PRELIMINARIES}

In this section, we briefly review some concepts and some related results of soft set, soft topological space and soft bitopological space which are needed to used in current paper. For more details about these concepts you can see [[10], [5], [6], [7], [8], [9], [11], [13], [14], [19], [20], [21], [25], [26], [3], [27]].

Let $X$ be an initial universe, $E$ be a set of parameters and $P(X)$ be the power set of $X$.

Definition 2.1 [21] A pair $(F, E)$ is called a soft set over $X$, where $F$ is a mapping given by $F: E \rightarrow P(X)$. A soft set can also be defined by the set of ordered pairs

$$
(F, E)=\{(e, F(e)): e \in E, F: E \rightarrow P(X)\} .
$$

From now on, $S S(X)_{E}$ denotes the family of all soft sets over $X$ with a fixed set of parameters $E$.

For two soft sets $(F, E),(G, E) \in S S(X)_{E},(F, E)$ is called a soft subset of $(G, E)$, denoted by $(F, E) \subseteq(G, E)$, if $F(e) \subseteq G(e), \forall e \in E$. In this case, $(G, E)$ is called a soft superset of $(F, E)$. In addition, the union of soft sets $(F, E)$ and $(G, E)$, denoted by $(F, E) \widetilde{\cup}(G, E)$, is the soft set $(H, E)$ which defined as $H(e)=F(e) \cup G(e), \forall e \in E$. Moreover, the intersection of soft sets $(F, E)$ and $(G, E)$ 
, denoted by $(F, E) \tilde{\cap}(G, E)$, is the soft set $(M, E)$ which defined as $\forall e \in E$. The complement of a soft set $(F, E)$, denoted by $(F, E)^{c}$, is defined as $(F, E)^{c}=\left(F^{c}, E\right)$, where $F^{c}: E \rightarrow P(X)$ is a mapping given by $F^{c}(e)=X \backslash F(e), \forall e \in E$. The difference of soft sets $(F, E)$ and $(G, E)$, denoted by $(F, E) \backslash(G, E)$, is the soft set $(H, E)$, which defined as $\quad H(e)=F(e) \backslash G(e), \quad \forall e \in E . \quad$ Clearly, $(F, E) \backslash(G, E)=(F, E) \tilde{\cap}(G, E)^{c}$. A soft set $(F, E)$ is called a null soft set, denoted by $(\tilde{\phi}, E)$, if $F(e)=\phi, \forall e \in E$. Moreover, a soft set $(F, E)$ is called an absolute soft set, denoted by $(\tilde{X}, E)$, if $F(e)=X, \quad \forall e \in E . \quad$ Clearly, we have $(\tilde{\phi}, E)^{c}=(\tilde{X}, E)$ and $(\tilde{X}, E)^{c}=(\tilde{\phi}, E)$. Moreover, a soft set $(G, E)$ is said to be a finite soft set if $G(e)$ is a finite set for all $e \in E$. Otherwise, it is called an infinite soft set. A soft set $(G, E)$ over $X$ is called a proper soft subset of $(\tilde{X}, E)$ if $(G, E) \subsetneq(\tilde{X}, E)$.

Definition 2.2 (,) A soft set $(F, E) \in S S(X)_{E}$ is said to be a soft point in $(\tilde{X}, E)$ if there exist $x \in X$ and $e \in E$ such that $F(e)=\{x\}$ and $F\left(e^{\prime}\right)=\phi$ for each $e^{\prime} \in E \backslash\{e\}$. This soft point is denoted by $\left(x_{e}, E\right)$ or $x_{e}$, i.e.

$$
\begin{gathered}
x_{e}: E \rightarrow P(X) \text { is a mapping defined as } \\
x_{e}(a)=\left\{\begin{array}{ll}
\{x\} & \text { if } e=a, \\
\phi & \text { if } e \neq a
\end{array} \text { for all } a \in E .\right.
\end{gathered}
$$

A soft point $\left(x_{e}, E\right)$ is said to be belonging to the soft set $(G, E)$, denoted by $x_{e} \tilde{\in}(G, E)$, if $x_{e}(e) \subseteq G(e)$, i.e., $\{x\} \subseteq G(e)$. Clearly, $x_{e} \tilde{\in}(G, E)$ if and only if $\left(x_{e}, E\right) \widetilde{\subseteq}(G, E)$. In addition, two soft points $x_{e_{1}}, y_{e_{2}}$ over $X$ are said to be equal if $x=y$ and $e_{1}=e_{2}$. Thus, $x_{e_{1}} \neq y_{e_{2}}$ iff $x \neq y$ or $e_{1} \neq e_{2}$.

The family of all soft points in $(\tilde{X}, E)$ is denoted by $\xi(X)_{E}$.

Proposition 2.1 [3] The union of any collection of soft points can be considered as a soft set and every soft set can be expressed as a union of all soft points belonging to it, i.e., $(G, E)=\bigcup\left\{\left(x_{e}, E\right): x_{e} \tilde{\in}(G, E)\right\}$.

Proposition 2.2 [3] Let $(G, E),(H, E)$ be two soft sets over $X$. Then,

(1) $x_{e} \tilde{\in}(G, E) \Leftrightarrow x_{e} \nsubseteq(G, E)^{c}$.

(2) $x_{e} \tilde{\in}(G, E) \widetilde{\cup}(H, E) \Leftrightarrow x_{e} \tilde{\in}(G, E)$ or $x_{e} \tilde{\in}(H, E)$.

(3) $x_{e} \tilde{\in}(G, E) \tilde{\cap}(H, E) \Leftrightarrow x_{e} \tilde{\in}(G, E)$ and $x_{e} \tilde{\in}(H, E)$.

(4) $(G, E) \simeq(H, E) \Leftrightarrow$ $\left[x_{e} \tilde{\in}(G, E) \Rightarrow x_{e} \tilde{\in}(H, E)\right]$.

For more details for soft point you can see [[20],[3],[22]].

Definition 2.3 [26], [23] Let $\eta$ be a collection of soft sets over a universe $X$ with a fixed set of parameters $E$, i.e., $\eta \subseteq S S(X)_{E}$. The collection $\eta$ is called a soft topology on $X$ if it satisfies the following axioms:

(1) $(\tilde{X}, E),(\tilde{\phi}, E) \in \eta$,

(2) The union of any number of soft sets in $\eta$ belongs to $\eta$,

(3) The intersection of any two soft sets in $\eta$ belongs to $\eta$.

The triple $(X, \eta, E)$ is called a soft topological space. Any member of $\eta$ is said to be an open soft set in $(X, \eta, E)$. A soft set $(F, E)$ over $X$ is said to be a closed soft set in $(X, \eta, E)$, if its complement $(F, E)^{c}$ is an open soft set in $(X, \eta, E)$. We denote the family of all closed soft sets by $\eta^{c}$.

Definition 2.4 [26] Let $(X, \eta, E)$ be a soft topological space and $(F, E) \in S S(X)_{E}$. The soft closure of $(F, E)$, denoted by $\operatorname{scl}_{\eta}(F, E)$, is defined by $\left.s c l_{\eta}(F, E)=\bigcap\left\{(H, E) \in \eta^{c}:(F, E) \subseteq(H, E)\right\}\right)$

Definition 2.5 [1] The two soft sets $(A, E),(B, E)$ in a soft topological space $(X, \eta, E)$ are called separated soft sets

$\operatorname{scl}_{\eta}(A, E) \tilde{\cap}(B, E)=\operatorname{scl}_{\eta}(B, E) \tilde{\cap}(A, E)=(\tilde{\phi}, E)$

.Definition 2.6 [1] A soft topological space $(X, \eta, E)$ is called soft connected space if $X$ cannot be expressed as a union of two separated soft sets. 
$s c l_{\eta}(A, E) \tilde{\cap}(B, E)=s c l_{\eta}(B, E) \tilde{\cap}(A, E)=(\tilde{\phi}, E)$

.Definition 2.7 [1] A soft set $(G, E)$ in a soft topological space $(X, \eta, E)$ is called a disconnected soft set if there exist two separated soft sets $(A, E),(B, E)$ such that $(G, E)=(A, E) \widetilde{\cup}(B, E)$. Otherwise, $\quad(G, E)$ is called a connected soft set.

Definition 2.8 [17],[27] Let $S S(X)_{E}$ and $S S(Y)_{K}$ be two families of soft sets. Let $u: X \rightarrow Y$ and $p: E \rightarrow K$ be mappings. We define a soft mapping $f_{p u}: S S(X)_{E} \rightarrow S S(Y)_{K}$ as:

(1) If $(G, E) \in S S(X)_{E}$. Then, the image of $(G, E)$ under $f_{p u}$, written as $f_{p u}(G, E)=\left(f_{p u}(G), p(E)\right)$, is a soft set in $S S(Y)_{K}$ such that

$f_{p u}(G, E)(k)=\left\{\begin{array}{lll}\bigcup_{e \in p^{-1}(k)} u[G(e)] & \text { if } & p^{-1}(k) \neq \phi, \\ \phi & \text { if } p^{-1}(k)=\phi\end{array}\right.$ for all $k \in K$.

(2) If $(H, K) \in S S(Y)_{K}$. Then, the inverse image of $(H, K)$ under $f_{p u}$, written as $f_{p u}^{-1}(H, K)=\left(f_{p u}^{-1}(H), E\right)$, is a soft set in $S S(X)_{E}$ such that

$f_{p u}^{-1}(H, K)(e)=u^{-1}[H(p(e))] \forall e \in E$.

Theorem 2.1 [17] Let $S S(X)_{E}$ and $S S(Y)_{K}$ be two families of soft sets. For a soft mapping

$f_{p u}: S S(X)_{E} \rightarrow S S(Y)_{K}$ we have the following:

(1) $f_{p u}(\tilde{\phi}, E)=(\tilde{\phi}, K)$ and $f_{p u}(\tilde{X}, E) \simeq(\tilde{Y}, K)$.

(2) $f_{p u}\left[\bigcup_{i \in I}\left(G_{i}, E\right)\right]=\bigcup_{i \in I} f_{p u}\left(G_{i}, E\right)$ and $f_{p u}\left[\bigcap_{i \in I}\left(G_{i}, E\right)\right] \simeq \bigcap_{i \in I} f_{p u}\left(G_{i}, E\right)$.

(3) $(G, E) \simeq(M, E) \Rightarrow f_{p u}(G, E) \simeq f_{p u}(M, E)$

. Theorem 2.2 [17],[18] Let $S S(X)_{E}$ and $S S(Y)_{K}$ be two families of soft sets. For a soft mapping $f_{p u}: S S(X)_{E} \rightarrow S S(Y)_{K}$ we have the following:

(1) $f_{p u}^{-1}(\tilde{\phi}, K)=(\tilde{\phi}, E)$ and $f_{p u}^{-1}(\tilde{Y}, K)=(\tilde{X}, E)$.
(2) $f_{p u}^{-1}\left[\bigcup_{i \in I}\left(H_{i}, K\right)\right]=\bigcup_{i \in I} f_{p u}^{-1}\left(H_{i}, K\right)$ and $f_{p u}^{-1}\left[\bigcap_{i \in I}\left(H_{i}, K\right)\right]=\bigcap_{i \in I} f_{p u}^{-1}\left(H_{i}, K\right)$.

(3) $(H, K) \simeq(N, K) \Rightarrow f_{p u}^{-1}(H, K) \simeq f_{p u}^{-1}(N, K)$

. Definition 2.9 [27] Let $S S(X)_{E}$ and $S S(Y)_{K}$ be two families of soft sets. A soft mapping

$f_{p u}: S S(X)_{E} \rightarrow S S(Y)_{K}$ is called soft surjective(soft injective) mapping if $u, p$ are surjective (injective) mappings, respectively. A soft mapping which is a soft surjective and soft injective mapping is called a soft bijection mapping.

Theorem 2.3 [27] Let $S S(X)_{E}$ and $S S(Y)_{K}$ be two families of soft sets. For a soft mapping

$f_{p u}: S S(X)_{E} \rightarrow S S(Y)_{K}$ we have the following:

(1) $f_{p u}^{-1}[(\tilde{Y}, K) \backslash(H, K)]=(\tilde{X}, E) \backslash\left[f_{p u}^{-1}(H, K)\right]$ for any $(H, K) \in S S(Y)_{K}$.

(2) $(G, E) \subseteq f_{p u}^{-1}\left[f_{p u}(G, E)\right]$ for any

$(G, E) \in S S(X)_{E}$. If $f_{p u}$ is a soft injective, the equality holds.

(3) $f_{p u}\left[f_{p u}^{-1}(H, K)\right] \simeq(H, K)$ for any $(H, K) \in S S(Y)_{K}$. If $f_{p u}$ is a soft surjective, the equality holds.

Definition 2.10 [28] Let $(X, \eta, E)$ and $(Y, \sigma, K)$ be two soft topological spaces. A soft mapping $f_{p u}:(X, \eta, E) \rightarrow(Y, \sigma, K)$ is said to be a soft continuous if the inverse image of any open soft set in $(Y, \sigma, K)$ is an open soft set in $(X, \eta, E)$, i.e., $f_{p u}^{-1}(H, K) \in \eta$ for any $(H, K) \in \sigma$.

Definition 2.11 [4] A triple $\left(X, \tau_{1}, \tau_{2}\right)$ is called a bitopological space [briefly, bts], where $\tau_{1}, \tau_{2}$ are arbitrary topologies on $X$. The collection $\tau_{12}$ is a supra topology on $X$, where

$$
\tau_{12}=\left\{G \subseteq X: G=G_{1} \cup G_{2} ; G_{i} \in \tau_{i}, i=1,2\right\}
$$

Definition 2.12 [4] Let $\left(X, \tau_{1}, \tau_{2}\right)$ be a bts. The sub $A, B$ of $X$ are said to be $p$-separated sets in $\left(X, \tau_{1}, \tau_{2}\right)$ if where $c l_{\tau_{12}}(A)=\left\{F \in \tau_{12}^{c}: A \subseteq F\right\}$.

Definition 2.13 [4] A bts $\left(X, \tau_{1}, \tau_{2}\right)$ is called $p$ disconnected if $X$ can be expressed as a union of two 
nonempty $p$-separated sets. Otherwise, $\left(X, \tau_{1}, \tau_{2}\right)$ is called $p$-connected.

Definition 2.14 [10] A quadrable system $\left(X, \eta_{1}, \eta_{2}, E\right)$ is called a soft bitopological space [briefly, sbts], where $\eta_{1}$, $\eta_{2}$ are arbitrary soft topologies on $X$ with a fixed set of parameters $E$.

Definition 2.15 [13] Let $\left(X, \eta_{1}, \eta_{2}, E\right)$ be a sbts. A soft set $(G, E)$ over $X$ is said to be a pairwise open soft set in $\left(X, \eta_{1}, \eta_{2}, E\right)$ [briefly, $p$-open soft set] if there exist an open soft set $\left(G_{1}, E\right)$ in $\eta_{1}$ and an open soft set $\left(G_{2}, E\right)$ in $\eta_{2}$ such that $(G, E)=\left(G_{1}, E\right) \widetilde{\cup}\left(G_{2}, E\right)$. A soft set $(G, E)$ over $X$ is said to be a pairwise closed soft set in $\left(X, \eta_{1}, \eta_{2}, E\right) \quad$ [briefly, $\quad p$-closed soft set] if its complement is a $p$-open soft set in $\left(X, \eta_{1}, \eta_{2}, E\right)$. Clearly, a soft set $(F, E)$ over $X$ is a $p$-closed soft set in $\left(X, \eta_{1}, \eta_{2}, E\right)$ if there exist an $\eta_{1}$-closed soft set $\left(F_{1}, E\right)$ and an $\eta_{2}$-closed soft set $\left(F_{2}, E\right)$ such that $(F, E)=\left(F_{1}, E\right) \widetilde{\cap}\left(F_{2}, E\right)$.

The family of all $p$-open ( $p$-closed) soft sets in a sbts $\left(X, \eta_{1}, \eta_{2}, E\right)$ is denoted by $\eta_{12}\left(\eta_{12}^{c}\right)$, respectively.

Theorem 2.4 [13] Let $\left(X, \eta_{1}, \eta_{2}, E\right)$ be a sbts. The family of all $p$-open soft sets $\eta_{12}$ is a supra soft topology on $X$, where

$\eta_{12}=\left\{\left(G_{1}, E\right) \widetilde{\cup}\left(G_{2}, E\right):\left(G_{i}, E\right) \in \eta_{i}, i=1,2\right\}$.

The triple $\left(X, \eta_{12}, E\right)$ is the supra soft topological space associated to the sbts $\left(X, \eta_{1}, \eta_{2}, E\right)$.

Lemma 2.1 [11] For any sbts $\left(X, \eta_{1}, \eta_{2}, E\right)$, we have $\left(X, \eta_{1}^{e}, \eta_{2}^{e}\right)$ is an ordinary bitopological space, for all $e \in E$, where $\eta_{i}^{e}=\left\{G(e):(G, E) \in \eta_{i}\right\}, i=1,2$, and $\eta_{12}^{e}=\eta_{12}(e)=\left\{H(e):(H, E) \in \eta_{12}\right\}$.

Definition 2.16 [13] Let $\left(X, \eta_{1}, \eta_{2}, E\right)$ be a sbts and let $(G, E) \in S S(X)_{E}$. The pairwise soft closure of $(G, E)$ , denoted by $\operatorname{scl}_{\eta_{12}}(G, E)$, is defined by $s c l_{\eta_{12}}(G, E)=\bar{\bigcap}\left\{(F, E) \in \eta_{12}^{c}:(G, E) \widetilde{\subseteq}(F, E)\right\}$ .Clearly, $s c l_{\eta_{12}}(G, E)$ is the smallest $p$-closed soft set contains $(G, E)$.
Theorem 2.5 [13] Let $\left(X, \eta_{1}, \eta_{2}, E\right)$ be a sbts and $(G, E) \in S S(X)_{E}$. Then,

$$
\begin{gathered}
x_{e} \tilde{\in} s c l_{\eta_{12}}(G, E) \Leftrightarrow\left(O_{x_{e}}, E\right) \tilde{\cap}(G, E) \neq(\tilde{\phi}, E), \\
\forall\left(O_{x_{e}}, E\right) \in \eta_{12}\left(x_{e}\right) \text { where }\left(O_{x_{e}}, E\right) \text { is any p- }
\end{gathered}
$$

open soft set contains $x_{e}$ and $\eta_{12}\left(x_{e}\right)$ is the family of all popen soft sets contains $x_{e}$.

For more details about the properties of pairwise soft closure operator see [13].

Definition 2.17 [13] Let $\left(X, \eta_{1}, \eta_{2}, E\right)$ be a sbts and let $(G, E) \in S S(X)_{E}$. The pairwise soft kernel of $(G, E)$ [briefly, $\left.\operatorname{sker}_{\eta_{12}}(G, E)\right]$, is the intersection of all $p$-open soft supersets of $(G, E)$, i.e.,

$\operatorname{sker}_{\eta_{12}}(G, E)=\bar{\bigcap}\left\{(H, E) \in \eta_{12}:(G, E) \subseteq(H, E)\right\}$

Definition 2.18 [13] A soft set $(G, E)$ is said to be a pairwise $\Lambda$ - soft set in a sbts $\left(X, \eta_{1}, \eta_{2}, E\right)$ [briefly, $p \Lambda$-soft set] if sker $_{\eta_{12}}(G, E)=(G, E)$.

Theorem 2.6 [13] Every $p$-open soft set is a $p \Lambda$-soft set. Theorem 2.7 [13] Let $\left(X, \eta_{1}, \eta_{2}, E\right)$ be a sbts. Then, the class of all $p \Lambda$-soft sets is an Alexandroff soft topology on $X$. This soft topology we denoted by $\eta_{p \Lambda}$. The triple $\left(X, \eta_{p \Lambda}, E\right)$ is the soft topological space associated to the sbts $\left(X, \eta_{1}, \eta_{2}, E\right)$, induced by the family of all $p \Lambda$-soft sets.

Theorem 2.8 [13] Let $\left(X, \eta_{1}, \eta_{2}, E\right)$ be a sbts. Then,

$$
\eta_{1} \cup \eta_{2} \subseteq \eta_{12} \subseteq \eta_{p \Lambda} \subseteq S S(X)_{E}
$$

Definition 2.19 [14] A sbts $\left(X, \eta_{1}, \eta_{2}, E\right)$ is said to be a pairwise soft $T_{1}^{*} \quad$ [briefly $\left.P S T_{1}^{*}\right]$ if for each $x_{\alpha}, y_{\beta} \in \xi(X)_{E} \quad$ with $\quad x_{\alpha} \neq y_{\beta}$, there exist $(G, E),(H, E) \in \eta_{12} \quad$ such that $x_{\alpha} \tilde{\in}(G, E)$, $y_{\beta} \notin(G, E) \quad$ and $\quad y_{\beta} \tilde{\in}(H, E), \quad x_{\alpha} \nsubseteq(H, E)$.

Theorem 2.9 [11] A sbts $\left(X, \eta_{1}, \eta_{2}, E\right)$ is a PST $T_{1}^{*}$ iff every soft point either $p$-open soft set or $p$-closed soft set.

Definition 2.20 [16] Let $\left(X, \eta_{1}, \eta_{2}, E\right)$ and $\left(Y, \sigma_{1}, \sigma_{2}, K\right)$ be two soft bitopological spaces. A soft mapping $f_{p u}:\left(X, \eta_{1}, \eta_{2}, E\right) \rightarrow\left(Y, \sigma_{1}, \sigma_{2}, K\right) \quad$ is said to be a pairwise soft continuous [briefly, $p$-soft 
continuous] if the inverse image of any $p$-open soft set in $\left(Y, \sigma_{1}, \sigma_{2}, K\right)$ is a $p$-open soft set in $\left(X, \eta_{1}, \eta_{2}, E\right)$, i.e., $f_{p u}^{-1}(H, K) \in \eta_{12}$ for any $(H, K) \in \sigma_{12}$.

Theorem 2.10 [13] Let $\left(X, \eta_{1}, \eta_{2}, E\right)$ be a sbts and let $Y \subseteq X$. Then, $\left(Y, \eta_{1 Y}, \eta_{2 Y}, E\right)$ is also soft bitopological space where

$\eta_{i Y}=\left\{(\tilde{Y}, E) \tilde{\cap}\left(G_{i}, E\right):\left(G_{i}, E\right) \in \eta_{i}\right\}, i=1,2$

and $\tilde{Y}(e)=Y, \forall e \in E$. This soft bitopological space we will called soft bitopological subspace of $\left(X, \eta_{1}, \eta_{2}, E\right)$.

Theorem 2.11 [16] Let $\left(X, \eta_{1}, \eta_{2}, E\right)$ and $\left(Y, \sigma_{1}, \sigma_{2}, K\right)$ be two soft bitopological spaces and let $f_{p u}:\left(X, \eta_{1}, \eta_{2}, E\right) \rightarrow\left(Y, \sigma_{1}, \sigma_{2}, K\right)$ be a soft mapping. Then, the following statements are equivalent:

(1) $f_{p u}$ is a $p$-soft continuous.

(2) $f_{p u}^{-1}(F, K) \in \eta_{12}^{c}$ for all $(F, K) \in \sigma_{12}^{c}$.

(3) $f_{p u}\left[s c l_{\eta_{12}}(G, E)\right] \simeq \widetilde{\subseteq} s l_{\sigma_{12}}\left[f_{p u}(G, E)\right]$ for any $(G, E) \in S S(X)_{E}$

(4) $s c l_{\eta_{12}}\left[f_{p u}^{-1}(H, K)\right] \subseteq f_{p u}^{-1}\left[s c l_{\sigma_{12}}(H, K)\right]$ for any $(H, K) \in S S(Y)_{K}$.

(5) $f_{p u}^{-1}\left[\operatorname{sint}_{\sigma_{12}}(H, K)\right] \widetilde{\subseteq} \operatorname{sint}_{\eta_{12}}\left[f_{p u}^{-1}(H, K)\right]$ for any $(H, K) \in S S(Y)_{K}$.

\section{PAIRWISE SEPARATED SOFT SETS}

Definition 3.1 Let $\left(X, \eta_{1}, \eta_{2}, E\right)$ be a sbts and let $(G, E),(H, E)$ are non-null soft sets over $X$. The soft sets $(G, E)$ and $(H, E)$ are said to be pairwise separated soft sets [briefly, separated soft sets] if $s c l_{\eta_{12}}(G, E) \tilde{\cap}(H, E)=(\tilde{\phi}, E)$, $(G, E) \tilde{\cap} s c l_{\eta_{12}}(H, E)=(\tilde{\phi}, E)$.

Proposition 3.1 Let $\left(X, \eta_{1}, \eta_{2}, E\right)$ be a sbts. Then, every $p$-separated soft sets are disjoint soft sets.

Remark 3.1 The converse of Proposition 3.1 may not be true as shown by the following example.

Example 3.1 Let $X=\{x, y, z, w\}, E=\left\{e_{1}, e_{2}\right\}$ and let

$$
\begin{gathered}
\eta_{1}=\{(\tilde{\phi}, E),(\tilde{X}, E),(G, E),(H, E)\} \\
\eta_{2}=\{(\tilde{\phi}, E),(\tilde{X}, E),(M, E),(N, E)\} \\
(G, E)=\left\{\left(e_{1},\{x, w\}\right),\left(e_{2},\{z, w\}\right)\right\}, \\
(H, E)=\left\{\left(e_{1},\{y, z\}\right),\left(e_{2},\{x, y\}\right)\right\}, \\
(M, E)=\left\{\left(e_{1},\{w\}\right),\left(e_{2},\{y, w\}\right)\right\}, \\
(N, E)=\left\{\left(e_{1},\{x, w\}\right),\left(e_{2}, X\right)\right\} .
\end{gathered}
$$

It is easy to verify that

$$
\begin{aligned}
\eta_{12}=\{(\tilde{\phi}, E),(\tilde{X}, E),(G, E),(H, E),(M, E), \\
\left.(N, E),\left(P_{1}, E\right),\left(P_{2}, E\right)\right\} \text { where } \\
\left(P_{1}, E\right)=\left\{\left(e_{1},\{x, w\}\right),\left(e_{2},\{y, z, w\}\right)\right\}, \\
\left(P_{2}, E\right)=\left\{\left(e_{1},\{y, z, w\}\right),\left(e_{2},\{x, y, w\}\right)\right\} .
\end{aligned}
$$

Therefore, the family of all $p$-closed soft sets is $\eta_{12}^{c}=\left\{(\tilde{\phi}, E),(\tilde{X}, E),(G, E)^{c},(H, E)^{c},(M, E)^{c}\right.$, $\left.(N, E)^{c},\left(P_{1}, E\right)^{c},\left(P_{2}, E\right)^{c}\right\}$

where

$$
\begin{aligned}
& (G, E)^{c}=\left\{\left(e_{1},\{y, z\}\right),\left(e_{2},\{x, y\}\right)\right\}, \\
& (H, E)^{c}=\left\{\left(e_{1},\{x, w\}\right),\left(e_{2},\{z, w\}\right)\right\}, \\
& (M, E)^{c}=\left\{\left(e_{1},\{x, y, z\}\right),\left(e_{2},\{x, z\}\right)\right\} \\
& (N, E)^{c}=\left\{\left(e_{1},\{y, z\}\right),\left(e_{2}, \phi\right)\right\}, \\
& \left(P_{1}, E\right)^{c}=\left\{\left(e_{1},\{y, z\}\right),\left(e_{2},\{x\}\right)\right\}, \\
& \left(P_{2}, E\right)^{c}=\left\{\left(e_{1},\{x\}\right),\left(e_{2},\{z\}\right)\right\} . \text { Now, let } \\
& \left(F_{1}, E\right)=\left\{\left(e_{1},\{x\}\right),\left(e_{2},\{x\}\right)\right\}, \\
& \left(F_{2}, E\right)=\left\{\left(e_{1},\{y\}\right),\left(e_{2},\{y\}\right)\right\} \text { and } \\
& \left(F_{3}, E\right)=\left\{\left(e_{1},\{x\}\right),\left(e_{2},\{w\}\right)\right\} .
\end{aligned}
$$

It is clear that $\left(F_{2}, E\right)$ and $\left(F_{3}, E\right)$ are $p$-separated soft sets. Although the soft sets $\left(F_{1}, E\right)$ and $\left(F_{2}, E\right)$ are disjoint, we find that their are not $p$-separated soft sets because

$$
s c l_{\eta_{12}}\left(F_{2}, E\right) \tilde{\cap}\left(F_{1}, E\right)=\left\{\left(e_{1}, \phi\right),\left(e_{2},\{x\}\right)\right\} \neq(\tilde{\phi}, E)
$$

Proposition 3.2 Let $\left(X, \eta_{1}, \eta_{2}, E\right)$ be a sbts and let $(G, E),(H, E)$ are non-null soft sets over $X$. If 
$s c l_{\eta_{12}}(G, E) \tilde{\cap} s c l_{\eta_{12}}(H, E)=(\tilde{\phi}, E)$,

then

$(G, E)$ and $(H, E)$ are $p$-separated soft sets.

Proof. Straightforward.

Note: From Propositions 3.1 and 3.2 we deduce that the concept of $p$-separated soft sets is a weaker than of the condition of disjoint pairwise soft closure of soft sets, but it is a stronger than of the concept of disjoint soft sets.

Remark 3.2 The converse of Proposition 3.2 may not be true as shown by the following example. Example 3.2 Let

$X=\{x, y, z\}, E=\left\{e_{1}, e_{2}\right\}$ and let

$$
\eta_{1}=\left\{(\tilde{\phi}, E),(\tilde{X}, E),\left(G_{1}, E\right),\left(G_{2}, E\right),\left(G_{3}, E\right)\right\},
$$

$\eta_{2}=\left\{(\tilde{\phi}, E),(\tilde{X}, E),\left(H_{1}, E\right),\left(H_{2}, E\right),\left(H_{3}, E\right)\right\}$

such that

$\left(G_{1}, E\right)=\left\{\left(e_{1},\{x, z\}\right),\left(e_{2},\{x, y\}\right)\right\}$,

$\left(G_{2}, E\right)=\left\{\left(e_{1},\{y, z\}\right),\left(e_{2},\{y, z\}\right)\right\}$

$\left(G_{3}, E\right)=\left\{\left(e_{1},\{z\}\right),\left(e_{2},\{y\}\right)\right\}$, and

$\left(H_{1}, E\right)=\left\{\left(e_{1},\{x, y\}\right),\left(e_{2},\{x, z\}\right)\right\}$,

$\left(H_{2}, E\right)=\left\{\left(e_{1},\{z\}\right),\left(e_{2},\{x, y\}\right)\right\}$,

$\left(H_{3}, E\right)=\left\{\left(e_{1}, \phi\right),\left(e_{2},\{x\}\right)\right\}$.

It is clear that $\left(X, \eta_{1}, \eta_{2}, E\right)$ is a sbts. Moreover,

$$
\eta_{12}=\left\{(\tilde{\phi}, E),(\tilde{X}, E),\left(G_{1}, E\right),\left(G_{2}, E\right),\left(G_{3}, E\right),\right.
$$$$
\left.\left(H_{1}, E\right),\left(H_{2}, E\right),\left(H_{3}, E\right),(P, E)\right\}
$$

where

$$
(P, E)=\left\{\left(e_{1},\{y, z\}\right),\left(e_{2}, X\right)\right\} .
$$

Consequently,

$$
\begin{gathered}
\eta_{12}^{c}=\left\{(\tilde{\phi}, E),(\tilde{X}, E),\left(G_{1}^{c}, E\right),\left(G_{2}^{c}, E\right),\left(G_{3}^{c}, E\right),\right. \\
\left.\left(H_{1}^{c}, E\right),\left(H_{2}^{c}, E\right),\left(H_{3}^{c}, E\right),\left(P^{c}, E\right)\right\}
\end{gathered}
$$

where

$$
\begin{aligned}
& \left(G_{1}^{c}, E\right)=\left\{\left(e_{1},\{y\}\right),\left(e_{2},\{z\}\right)\right\}, \\
& \left(G_{2}^{c}, E\right)=\left\{\left(e_{1},\{x\}\right),\left(e_{2},\{x\}\right)\right\}, \\
& \left(G_{3}^{c}, E\right)=\left\{\left(e_{1},\{x, y\}\right),\left(e_{2},\{x, z\}\right)\right\}, \\
& \left(H_{1}^{c}, E\right)=\left\{\left(e_{1},\{z\}\right),\left(e_{2},\{y\}\right)\right\}, \\
& \left(H_{2}^{c}, E\right)=\left\{\left(e_{1},\{x, y\}\right),\left(e_{2},\{z\}\right)\right\}, \\
& \left(H_{3}^{c}, E\right)=\left\{\left(e_{1}, X\right),\left(e_{2},\{y, z\}\right)\right\}, \\
& \left(P^{c}, E\right)=\left\{\left(e_{1},\{x\}\right),\left(e_{2}, \phi\right)\right\}
\end{aligned}
$$

Now, let

$$
\begin{aligned}
& (M, E)=\left\{\left(e_{1},\{y\}\right),\left(e_{2},\{y\}\right)\right\} \text { and } \\
& (N, E)=\left\{\left(e_{1}, \phi\right),\left(e_{2},\{x\}\right)\right\} . \text { Then, }
\end{aligned}
$$

$\operatorname{scl}_{12}(M, E)=\left(H_{3}^{c}, E\right)$ and $\operatorname{scl}_{12}(N, E)=\left(G_{2}^{c}, E\right)$ . It is clear that $(M, E),(N, E)$ are $p$-separated soft sets but $\quad \operatorname{scl}_{12}(M, E) \tilde{\cap} s c l_{12}(N, E)=$ $\left\{\left(e_{1},\{x\}\right),\left(e_{2}, \phi\right)\right\} \neq(\tilde{\phi}, E)$.

Proposition 3.3 Let $\left(X, \eta_{1}, \eta_{2}, E\right)$ be a sbts. If $(G, E)$, $(H, E)$ are $p$-separated soft sets, $(M, E) \widetilde{\subseteq}(G, E)$ and $(N, E) \widetilde{\subseteq}(H, E)$, then $(M, E),(N, E)$ are also $p$-separated soft sets.

Proof. From Theorem 3.11 in [13], we have $s c l_{\eta_{12}}(M, E) \simeq s c l_{\eta_{12}}(G, E)$. Since $(N, E) \simeq(H, E)$, then $s c l_{\eta_{12}}(M, E) \tilde{\cap}(N, E) \tilde{\subseteq} s c l_{\eta_{12}}(G, E) \tilde{\cap}(H, E)=(\tilde{\phi}, E)$ Therefore, $s c l_{\eta_{12}}(M, E) \tilde{\cap}(N, E)=(\tilde{\phi}, E)$. ву similar we can prove that $s c l_{\eta_{12}}(N, E) \tilde{\cap}(M, E)=(\tilde{\phi}, E)$. Hence, $(M, E),(N, E)$ are $p$-separated soft sets.

Proposition 3.4 Let $\left(X, \eta_{1}, \eta_{2}, E\right)$ be a sbts and let $(G, E)$ and $(H, E)$ are $p$-open soft sets. Then, $(G, E)$ and $(H, E)$ are $p$-separated soft sets if and only if $(G, E)$ and $(H, E)$ are disjoint. Proof. The first direction is obvious from Proposition 3.1. Now, suppose that $(G, E)$ and $(H, E)$ are disjoint $p$-open soft sets, then $(G, E) \widetilde{\subseteq}(H, E)^{c} \in \eta_{12}^{c}$. It follows that $s c l_{\eta_{12}}(G, E) \widetilde{\subseteq}(H, E)^{c}$ implies $s c l_{\eta_{12}}(G, E) \tilde{\cap}(H, E)=(\tilde{\phi}, E)$. By similar way we can show that $s c l_{\eta_{12}}(H, E) \tilde{\cap}(G, E)=(\tilde{\phi}, E)$. Hence, $(G, E),(H, E)$ are $p$-separated soft sets.

Proposition 3.5 Let $\left(X, \eta_{1}, \eta_{2}, E\right)$ be a sbts and let $(F, E)$ and $(M, E)$ are $p$-closed soft sets. Then, $(F, E)$ and $(M, E)$ are $p$-separated soft sets if and only if $(F, E)$ and $(M, E)$ are disjoint.

Proof. Straightforward. 
Theorem 3.1 Let $\left(X, \eta_{1}, \eta_{2}, E\right)$ be a $P S T_{1}^{*}$ and let $(G, E),(H, E)$ be two finite and disjoint soft sets. Then $(G, E)$ and $(H, E)$ are $p$-separated soft sets.

Proof. Since $\left(X, \eta_{1}, \eta_{2}, E\right)$ is a $P S T_{1}^{*}$, then every soft point is a $p$-closed soft set. Therefore, every finite soft set is a $p$-closed soft set. Since $(G, E)$ and $(H, E)$ are finite soft sets, then $(G, E)$ and $(H, E)$ are $p$-closed soft sets. It follows by Proposition 3.5 that $(G, E)$ and $(H, E)$ are $p$-separated soft sets.

Theorem 3.2 Let $\left(X, \eta_{1}, \eta_{2}, E\right)$ be a sbts, $Y \subseteq X$ and let $(G, E),(H, E) \widetilde{\subseteq}(\tilde{Y}, E) \widetilde{\subseteq}(\tilde{X}, E)$. Then,

if $(G, E),(H, E)$ are $p$-separated soft sets in

$\left(X, \eta_{1}, \eta_{2}, E\right)$, then their are $p$-separated soft sets in

$\left(Y, \eta_{1 Y}, \eta_{2 Y}, E\right)$ where

$\eta_{i Y}=\left\{(\tilde{Y}, E) \tilde{\cap}(G, E):(G, E) \in \eta_{i}\right\}, i=1,2$.

Proof. $s c l_{\eta_{12 Y}}(G, E)=$

$\bigcap\left\{(F, E):(F, E) \in \eta_{12 Y}^{c}:(G, E) \simeq(F, E)\right\}$

$=\bigcap\left\{(\tilde{Y}, E) \tilde{\cap}(M, E):(M, E) \in \eta_{12}^{c},(G, E) \subseteq\right.$ $(\tilde{Y}, E) \tilde{\cap}(M, E)\}$

$\widetilde{\subseteq}\left\{(\tilde{Y}, E) \tilde{\cap}(M, E):(M, E) \in \eta_{12}^{c}:(G, E) \widetilde{\subseteq}\right.$ $(M, E)\}$

$=(\tilde{Y}, E) \tilde{\cap}\left[\bigcap\left\{(M, E):(M, E) \in \eta_{12}^{c}:(G, E) \widetilde{\subseteq}\right.\right.$ $(M, E)\}]=(\tilde{Y}, E) \tilde{\cap} s c l_{\eta_{12}}(G, E)$. Hence,

$s c l_{\eta_{12 Y}}(G, E) \widetilde{\subseteq}(\tilde{Y}, E) \tilde{\cap} s c l_{\eta_{12}}(G, E)$. It follows

that $s c l_{\eta_{12 Y}}(G, E) \tilde{\cap}(H, E) \widetilde{\subseteq}(\tilde{Y}, E) \tilde{\cap}(H, E)$ $\tilde{\cap} s c l_{\eta_{12}}(G, E)$. Therefore,

$s c l_{\eta_{12 Y}}(G, E) \tilde{\cap}(H, E) \widetilde{\subseteq}$

$(H, E) \tilde{\cap} s c l_{\eta_{12}}(G, E)=(\tilde{\phi}, E)$. Consequently,

$s c l_{\eta_{12 Y}}(G, E) \tilde{\cap}(H, E)=(\widetilde{\phi}, E)$. By similar way we can prove that $s c l_{\eta_{12 Y}}(H, E) \tilde{\cap}(H, E)=(\tilde{\phi}, E)$.

Hence, $(G, E),(H, E)$ are $p$-separated soft sets in $\left(Y, \eta_{1 Y}, \eta_{2 Y}, E\right)$.

Theorem 3.3 Let

$f_{p u}:\left(X, \eta_{1}, \eta_{2}, E\right) \rightarrow\left(Y, \sigma_{1}, \sigma_{2}, K\right)$ be a $p$-soft continuous and soft surjective mapping. If

$(M, K),(N, K)$ are $p$-separated soft sets in

$\left(Y, \sigma_{1}, \sigma_{2}, K\right)$, then $f_{p u}^{-1}(M, K), f_{p u}^{-1}(N, K)$ are $p$ -separated soft sets in $\left(X, \eta_{1}, \eta_{2}, E\right)$.

Proof. Since $(M, K),(N, K)$ are $p$-separated soft sets in $\left(Y, \sigma_{1}, \sigma_{2}, K\right)$, then

$\operatorname{scl}_{\sigma_{12}}(M, K) \tilde{\cap}(N, K)=(\tilde{\phi}, K)$ and

$(M, K) \tilde{\cap} s c l_{\sigma_{12}}(N, K)=(\tilde{\phi}, K)$. Since $f_{p u}$ is a $p$-soft continuous mapping, then we deduce by Theorem 2.11 that $s c l_{\eta_{12}}\left[f_{p u}^{-1}(M, K)\right] \subseteq f_{p u}^{-1}\left[s c l_{\sigma_{12}}(M, K)\right]$ which implies that $f_{p u}^{-1}(N, K) \tilde{\cap} s c l_{\eta_{12}}\left[f_{p u}^{-1}(M, K)\right] \widetilde{\subseteq}$ $f_{p u}^{-1}(N, K) \tilde{\cap} f_{p u}^{-1}\left[s c l_{\sigma_{12}}(M, K)\right]$ $=f_{p u}^{-1}\left[(N, K) \tilde{\cap} s c l_{\sigma_{12}}(M, K)\right]$ [by Theorem 2.2(2)] $=f_{p u}^{-1}[(\tilde{\phi}, K)]$ $=(\tilde{\phi}, E)$ [by Theorem $2.2(1)]$.

Hence, $f_{p u}^{-1}(N, K) \tilde{\cap} s c l_{\eta_{12}}\left[f_{p u}^{-1}(M, K)\right]=(\tilde{\phi}, E)$ Similarly, we can prove that $f_{p u}^{-1}(M, K) \tilde{\cap} s c l_{\eta_{12}}\left[f_{p u}^{-1}(N, K)\right]=(\tilde{\phi}, E)$. Since $f_{p u}$ is a soft surjective mapping, then $f_{p u}^{-1}(M, K) \neq(\tilde{\phi}, E)$ and $f_{p u}^{-1}(N, K) \neq(\tilde{\phi}, E)$. Consequently, $f_{p u}^{-1}(M, K), f_{p u}^{-1}(N, K)$ are $p$ separated soft sets in $\left(X, \eta_{1}, \eta_{2}, E\right)$.

Theorem 3.4 Let $\left(X, \eta_{1}, \eta_{2}, E\right)$ be a sbts. Then, if $(G, E),(H, E)$ are $p$-separated soft sets in $\left(X, \eta_{1}, \eta_{2}, E\right)$, then $G(e), H(e)$ are $p$-separated sets in $\left(X, \eta_{1}^{e}, \eta_{2}^{e}\right)$, for all $e \in E$.

Proof. For any sbts $\left(X, \eta_{1}, \eta_{2}, E\right), e \in E$ we have $\eta_{12}^{e}=\eta_{12}(e)=\left\{G(e):(G, E) \in \eta_{12}\right\}$ [by Lemma 2.1]. Since $s c l_{\eta_{12}}(G, E)=\bigcap\left\{(F, E) \in \eta_{12}^{c}:(G, E) \simeq(F, E)\right\}$ , then $s c l_{\eta_{12}}(G, E)(e)=\bigcap\left\{F(e) \in \eta_{12}^{c}(e): G(e) \subseteq F(e)\right\}$ 
. Therefore, $s c l_{\eta_{12}}(G, E)(e)=c l_{\eta_{12}^{e}} G(e)$. Now, since $(G, E),(H, E)$ are $p$-separated soft sets in $\left(X, \eta_{1}, \eta_{2}, E\right)$, then $s c l_{\eta_{12}}(G, E) \tilde{\cap}(H, E)=(\tilde{\phi}, E)$ and $s c l_{\eta_{12}}(H, E) \tilde{\cap}(G, E)=(\tilde{\phi}, E)$ implies $\left[s c l_{\eta_{12}}(G, E) \tilde{\cap}(H, E)\right](e)=\phi$ and $\left[s c l_{\eta_{12}}(H, E) \tilde{\cap}(G, E)\right](e)=\phi$. Therefore, $s c l_{\eta_{12}} G(e) \cap H(e)=\phi$ and $s c l_{\eta_{12}} H(e) \cap G(e)=\phi$. It follows by Definition 2.13 that, $G(e), H(e)$ are $p$-separated sets in $\left(X, \eta_{1}^{e}, \eta_{2}^{e}\right)$.

Theorem 3.5 Let $\left(X, \eta_{1}, \eta_{2}, E\right)$ be a sbts. Then,

If $(G, E),(H, E)$ are $p$-separated soft sets in $\left(X, \eta_{1}, \eta_{2}, E\right)$, then their are soft separated sets in $\left(X, \eta_{p \Lambda}, E\right)$.

Proof. It follows from the fact that $s c l_{\eta_{p \Lambda}}(G, E) \subseteq s c l_{\eta_{12}}(G, E), \forall(G, E) \in S S(X)_{E}$

\section{PAIRWISE SOFT DISCONNECTED (CONNECTED) SPACES}

Definition 4.1 Let $\left(X, \eta_{1}, \eta_{2}, E\right)$ be a sbts. A $p$ separated soft sets $(G, E),(H, E)$ in $\left(X, \eta_{1}, \eta_{2}, E\right)$ are said to be pairwise soft separation of $(\tilde{X}, E)$ [briefly, $p$-soft separation] if $(\tilde{X}, E)=(G, E) \tilde{\cup}(H, E)$. In this case we say that $(\tilde{X}, E)$ has a $p$-soft separation. Definition 4.2 A sbts $\left(X, \eta_{1}, \eta_{2}, E\right)$ is said to be a pairwise soft disconnected space [briefly, $\quad p$-soft disconnected] if $(\tilde{X}, E)$ has a $p$-soft separation. Otherwise, $\left(X, \eta_{1}, \eta_{2}, E\right)$ is called pairwise soft connected space [briefly, $p$-soft connected], i.e., a sbts $\left(X, \eta_{1}, \eta_{2}, E\right)$ is a $p$-soft connected if $(\tilde{X}, E)$ cannot be represented as the union of two $p$-separated soft sets.

Example 4.1 From Example 3.1, $\left(X, \eta_{1}, \eta_{2}, E\right)$ is a $p$ soft disconnected because $(G, E)$ and $(H, E)$ are form a $p$-soft separation of $(X, E)$

where

$(G, E)=\left\{\left(e_{1},\{x, w\}\right),\left(e_{2},\{z, w\}\right)\right\}$,

$(H, E)=\left\{\left(e_{1},\{y, z\}\right),\left(e_{2},\{x, y\}\right)\right\}$.
Also, From Example 3.2, we deduce that $\left(X, \eta_{1}, \eta_{2}, E\right)$ is a $p$-soft disconnected because $\left(G_{3}, E\right)$ and $\left(H_{1}, E\right)$ are form a $p$-soft separation of $(X, E)$.

Proposition 4.1 Let $\left(X, \eta_{1}, \eta_{2}, E\right)$ be a sbts. Then,

(1) If $\eta_{1}=\eta_{2}=\{(\tilde{\phi}, E),(\tilde{X}, E)\}$, then

$\left(X, \eta_{1}, \eta_{2}, E\right)$ is a $p$-soft connected.

(2) If $\eta_{12}=S S(X)_{E},|X|>1$, then $\left(X, \eta_{1}, \eta_{2}, E\right)$ is a $p$-soft disconnected.

(3) If $\eta_{12}=\{(\tilde{\phi}, E),(\tilde{X}, E),(G, E)\}$, then $\left(X, \eta_{1}, \eta_{2}, E\right)$ is a $p$-soft connected.

Proof. (1): By given we deduce that $\eta_{12}=\{(\tilde{\phi}, E),(\tilde{X}, E)\}$. So, for any two soft sets $(G, E),(H, E) \quad$ over $\quad X \quad$ we have $s c l_{\eta_{12}}(G, E)=s c l_{\eta_{12}}(H, E)=(\tilde{X}, E)$.

Consequently, we cannot represented $(\tilde{X}, E)$ as a union of two $p$-separated soft sets in $\left(X, \eta_{1}, \eta_{2}, E\right)$. Hence, $\left(X, \eta_{1}, \eta_{2}, E\right)$ is a $p$-soft connected.

(2): Since $\eta_{12}=S S(X)_{E},|X|>1$, then every soft set is a $p$-closed soft set. It follows that for every soft point $\left(x_{e}, E\right)$ in $(\tilde{X}, E)$ we have $s c l_{\eta_{12}}\left(x_{e}, E\right)=\left(x_{e}, E\right)$ and $s c l_{\eta_{12}}\left(x_{e}, E\right)^{c}=\left(x_{e}, E\right)^{c}$. So, $\left(x_{e}, E\right),\left(x_{e}, E\right)^{c}$ are $\quad p$-separated soft sets and $(\tilde{X}, E)=\left(x_{e}, E\right) \tilde{\cup}\left(x_{e}, E\right)^{c}$. Hence, $\left(X, \eta_{1}, \eta_{2}, E\right)$ is a $p$-soft disconnected.

(3): Assume that $\left(X, \eta_{1}, \eta_{2}, E\right)$ is a $p$-soft disconnected. Then there exist two non-null soft sets $(M, E),(N, E)$ such that $\operatorname{scl}_{12}(M, E) \tilde{\cap}(N, E)=\operatorname{scl}_{12}(N, E) \tilde{\cap}(M, E)$ $=(\tilde{\phi}, E)$ and $(\tilde{X}, E)=(M, E) \tilde{\cup}(N, E)$. Therefore, $\operatorname{scl}_{12}(M, E)=\operatorname{scl}_{12}(N, E)=(G, E)^{c}$. It follows that $(M, E) \simeq(G, E)$ and $(N, E) \tilde{\subseteq}(G, E)$ implies $(M, E) \tilde{\cup}(N, E) \tilde{\subseteq}(G, E)$. Thus, $(\tilde{X}, E)=(G, E)$, a contradicts with that $(\tilde{X}, E) \neq(G, E)$. Hence, $\left(X, \eta_{1}, \eta_{2}, E\right)$ is a $p$-soft connected space. 
Theorem 4.1 Let $\left(X, \eta_{1}, \eta_{2}, E\right)$ be a sbts. Then the following are equivalent:

(1) $\left(X, \eta_{1}, \eta_{2}, E\right)$ is $p$-soft connected.

(2) $(\tilde{X}, E)$ cannot represented as a union of two non-null disjoint $p$-open soft sets.

(3) $(\tilde{X}, E)$ cannot represented as a union of two non null disjoint $p$-closed soft sets.

(4) $(\tilde{X}, E)$ has no proper soft subset which is both $p$-open and $p$-closed soft set other than $(\tilde{\phi}, E)$.

Proof. (1) $\Rightarrow(2)$ : Assume that there exist two non-null $p$-open soft sets $(G, E),(H, E)$ such that

$(G, E) \tilde{\cap}(H, E)=(\tilde{\phi}, E)$ and

$(\tilde{X}, E)=(G, E) \widetilde{\cup}(H, E)$. Since

$(G, E) \tilde{\cap}(H, E)=(\tilde{\phi}, E)$, then $(G, E) \widetilde{\subseteq}(H, E)^{c}$ , $(H, E) \simeq(G, E)^{c}$. Therefore,

$s c l_{\eta_{12}}(G, E) \tilde{\cap}(H, E)=(\tilde{\phi}, E)$ and

$\operatorname{scl}_{\eta_{12}}(H, E) \tilde{\cap}(G, E)=(\tilde{\phi}, E)$. It follows that

$(\tilde{X}, E)$ has a $p$-soft separation, i.e., $\left(X, \eta_{1}, \eta_{2}, E\right)$ is $p$-soft connected which contradicts with (1) .

$(2) \Rightarrow(3)$ : Assume that there exist two non-null $p$ closed soft sets $(F, E),(M, E)$ such that

$(F, E) \tilde{\cap}(M, E)=(\tilde{\phi}, E)$ and

$(\tilde{X}, E)=(F, E) \sim(M, E)$. Then,

$(F, E)^{c},(M, E)^{c}$ are non-null $p$-open soft sets and

$(F, E)^{c} \widetilde{\cup}(M, E)^{c}=(\tilde{X}, E)$, which contradicts with (2).

$(3) \Rightarrow(4)$ : Assume that there exists $(G, E) \subsetneq$ $(\tilde{X}, E),(N, E) \neq(\tilde{\phi}, E)$ such that $(N, E)$ is both $p$-open and $p$-closed soft set. Then $(N, E),(N, E)^{c}$ are non-null disjoint $p$-closed soft sets and

$(\tilde{X}, E)=(N, E) \widetilde{\cup}(N, E)^{c}$, this contradicts with (3)

.(4) $\Rightarrow(1)$ : Assume that $\left(X, \eta_{1}, \eta_{2}, E\right)$ is a $p$-soft disconnected. Then there exists $p$-separated soft sets

$(G, E),(H, E)$ such that

$(\tilde{X}, E)=(G, E) \widetilde{\cup}(H, E)$. Therefore,

$(G, E)^{c} \tilde{\cap}(H, E)^{c}=(\tilde{\phi}, E)$ implies
$(H, E)^{c} \widetilde{\subseteq}(G, E)$ and $(G, E)^{c} \widetilde{\subseteq}(H, E)$. Since $s c l_{\eta_{12}}(G, E) \tilde{\cap}(H, E)=(\tilde{\phi}, E)$, then $s c l_{\eta_{12}}(G, E) \widetilde{\subseteq}(H, E)^{c} \simeq(G, E)$. Hence, $(G, E)$ is $p$-closed soft set. Similarly, $(H, E)$ is $p$-closed soft set. On the other hand, by Proposition 3.1 we deduce that $(G, E) \simeq(H, E)^{c}$. Therefore, $(G, E)=(H, E)^{c}$. It follows that $(H, E)^{c}$ is $p$-closed soft set. Hence, $(H, E)$ is non-null $p$-closed and $p$-open soft set, which contradicts with (4).

Corollary 4.1 A sbts $\left(X, \eta_{1}, \eta_{2}, E\right)$ is a $p$-soft connected space if and only if the only soft sets over $X$ which are $p$-open and $p$-closed soft sets are $(\tilde{X}, E)$ and $(\tilde{\phi}, E)$.

Example 4.2 Let $X=\{a, b, c, d\}, E=\left\{e_{1}, e_{2}, e_{3}\right\}$ and let

$\eta_{1}=\left\{(\tilde{\phi}, E),(\tilde{X}, E),\left(G_{1}, E\right),\left(G_{2}, E\right)\right.$,

$\left.\left(G_{3}, E\right),\left(G_{4}, E\right)\right\}$

$\eta_{2}=\left\{(\tilde{\phi}, E),(\tilde{X}, E),\left(H_{1}, E\right),\left(H_{2}, E\right)\right\}$

where,

$\left(G_{1}, E\right)=\left\{\left(e_{1},\{a, c\}\right),\left(e_{2},\{a, b, c\}\right),\left(e_{3},\{c, d\}\right)\right\}$,

$\left(G_{2}, E\right)=\left\{\left(e_{1}, \phi\right),\left(e_{2},\{a, c\}\right),\left(e_{3},\{d\}\right)\right\}$,

$\left(G_{3}, E\right)=\left\{\left(e_{1},\{c\}\right),\left(e_{2},\{b\}\right),\left(e_{3}, \phi\right)\right\}$,

$\left(G_{4}, E\right)=\left\{\left(e_{1},\{c\}\right),\left(e_{2},\{a, b, c\}\right),\left(e_{3},\{d\}\right)\right\}$,

$\left(H_{1}, E\right)=\left\{\left(e_{1},\{a, b\}\right),\left(e_{2},\{a, c\}\right),\left(e_{3},\{a, d\}\right)\right\}$,

$\left(H_{2}, E\right)=\left\{\left(e_{1},\{b\}\right),\left(e_{2},\{c\}\right),\left(e_{3},\{a, d\}\right)\right\}$.

Then, $\left(X, \eta_{1}, \eta_{2}, E\right)$ is a sbts and therefore

$\eta_{12}=\left\{(\tilde{\phi}, E),(\tilde{X}, E),\left(G_{1}, E\right),\left(G_{2}, E\right),\left(G_{3}, E\right)\right.$,

$\left.,\left(G_{4}, E\right),\left(H_{1}, E\right),\left(H_{2}, E\right),\left(P_{1}, E\right),\left(P_{2}, E\right),\left(P_{3}, E\right),\left(P_{4}, E\right),\left(P_{5}, E\right)\right\}$

where

$\left(P_{1}, E\right)=\left\{\left(e_{1},\{a, b, c\}\right),\left(e_{2},\{a, b, c\}\right),\left(e_{3},\{a, c, d\}\right)\right\}$

,$\left(P_{2}, E\right)=\left\{\left(e_{1},\{b\}\right),\left(e_{2},\{a, c\}\right),\left(e_{3},\{a, d\}\right)\right\}$, 
$\left(P_{3}, E\right)=\left\{\left(e_{1},\{a, b, c\}\right),\left(e_{2},\{a, b, c\}\right),\left(e_{3},\{a, d\}\right)\right\}$

,$\left(P_{4}, E\right)=\left\{\left(e_{1},\{b, c\}\right),\left(e_{2},\{b, c\}\right),\left(e_{3},\{a, d\}\right)\right\}$,

$\left(P_{5}, E\right)=\left\{\left(e_{1},\{b, c\}\right),\left(e_{2},\{a, b, c\}\right),\left(e_{3},\{a, d\}\right)\right\}$.

It is easily to seen that the only soft sets over $X$ which are $p$-open and $p$-closed soft sets are $(\tilde{X}, E)$ and $(\tilde{\phi}, E)$. Consequently, by Corollary 4.1 we deduce that $\left(X, \eta_{1}, \eta_{2}, E\right)$ is a $p$-soft connected space.

Theorem 4.2 Let $\left(X, \eta_{1}, \eta_{2}, E\right)$ be a sbts. Then the following are equivalent:

(1) $\left(X, \eta_{1}, \eta_{2}, E\right)$ is $p$-soft disconnected.

(2) $(\tilde{X}, E)$ can represented as a union of two non-null disjoint $p$-open soft sets.

(3) $(\tilde{X}, E)$ can represented as a union of two non null disjoint $p$-closed soft sets.

(4) $(\tilde{X}, E)$ has a proper soft subset which is both $p$-open and $p$-closed soft set other than $(\tilde{\phi}, E)$.

Proof. Similar to the prove of Theorem 4.1.

Remark 4.1 Let $\left(X, \eta_{1}, \eta_{2}, E\right)$ be a $p$-soft connected space and let $e \in E$. Then $\left(X, \eta_{1}^{e}, \eta_{2}^{e}\right)$ may not be $p$ connected space as shown in the following example.

Example 4.3 Let $X=\{a, b, c\}, E=\left\{e_{1}, e_{2}\right\}$ and let

$$
\begin{aligned}
& \eta_{1}=\{(\tilde{\phi}, E),(\tilde{X}, E),(G, E)\}, \\
& \eta_{2}=\{(\tilde{\phi}, E),(\tilde{X}, E),(H, E)\},
\end{aligned}
$$

Where,

$$
\begin{aligned}
& (G, E)=\left\{\left(e_{1},\{a\}\right),\left(e_{2},\{b, c\}\right)\right\} \\
& (H, E)=\left\{\left(e_{1},\{b, c\}\right),\left(e_{2},\{a, c\}\right)\right\} .
\end{aligned}
$$

Then, $\left(X, \eta_{1}, \eta_{2}, E\right)$ is a sbts. It is Clear that $\eta_{12}=\{(\tilde{\phi}, E),(\tilde{X}, E),(G, E),(H, E)\}$. Therefore, $\left(X, \eta_{1}, \eta_{2}, E\right)$ is a $p$-soft connected because we cannot represented $(\tilde{X}, E)$ as a union of two non-null disjoint $p$ open soft sets. On the other hand, $\eta_{12}\left(e_{1}\right)=\left\{(\phi, X,\{a\},\{b, c\}\}\right.$. Hence, $\left(X, \eta_{1}^{e_{1}}, \eta_{2}^{e_{1}}\right)$ is a $p$-disconnected space because $\{a\}$ is both $p$-open and $p$-closed set.
Remark 4.2 Let $\left(X, \eta_{1}, \eta_{2}, E\right)$ be a $p$-soft disconnected space and let $e \in E$. Then $\left(X, \eta_{1}^{e}, \eta_{2}^{e}\right)$ may not be $p$ disconnected space as shown in the following example.

Example 4.4 Let $X=\{a, b, c\}, E=\left\{e_{1}, e_{2}\right\}$ and let

$$
\begin{aligned}
& \eta_{1}=\{(\tilde{\phi}, E),(\tilde{X}, E),(M, E),(N, E)\} \\
& \eta_{2}=\{(\tilde{\phi}, E),(\tilde{X}, E),(K, E)\},
\end{aligned}
$$

Where,

$$
\begin{aligned}
& (M, E)=\left\{\left(e_{1},\{b\}\right),\left(e_{2},\{b\}\right)\right\}, \\
& (N, E)=\left\{\left(e_{1}, X\right),\left(e_{2},\{b\}\right)\right\}, \text { and } \\
& (K, E)=\left\{\left(e_{1}, \phi\right),\left(e_{2},\{a, c\}\right)\right\} .
\end{aligned}
$$

Then, $\left(X, \eta_{1}, \eta_{2}, E\right)$ is a sbts. It is Clear that $\eta_{12}=\{(\tilde{\phi}, E),(\tilde{X}, E),(M, E),(N, E),(K, E),(P, E)\}$ where, $\quad(P, E)=\left\{\left(e_{1},\{b\}\right),\left(e_{2}, X\right)\right\}$. Since $\left\{\left(e_{1}, X\right),\left(e_{2},\{b\}\right)\right\}$ is both $p$-open and $p$-closed soft set, then by Theorem 4.2 (4) we have $\left(X, \eta_{1}, \eta_{2}, E\right)$ is a $p$-soft disconnected.

Now, $\eta_{1}^{e_{1}}=\{\phi,\{b\}, X\}$ and $\eta_{2}^{e_{1}}=\{\phi, X\}$.

So, $\eta_{12}^{e_{1}}=\{\phi, X,\{b\}\}$. Obvious that $\left(X, \eta_{1}^{e_{1}}, \eta_{2}^{e_{1}}\right)$ is a $p$-connected space. We can show that $\left(X, \eta_{1}^{e_{2}}, \eta_{2}^{e_{2}}\right)$ is a $p$-disconnected space.

Theorem 4.3 Let $\left(X, \eta_{1}, \eta_{2}, E\right)$ be sbts and let $Y \subseteq X$ .Then, $\left(Y, \eta_{1 Y}, \eta_{2 Y}, E\right)$ is a $p$-soft disconnected if and only if there exist two $p$-separated soft sets $\left(F^{Y}, E\right)$ and $\left(K^{Y}, E\right)$ in $\left(Y, \eta_{1 Y}, \eta_{2 Y}, E\right)$ such that $(\tilde{Y}, E)=\left(F^{Y}, E\right) \widetilde{\cup}\left(K^{Y}, E\right)$, where $\left(F^{Y}, E\right)=(\tilde{Y}, E) \tilde{\cap}(F, E),(F, E) \in \eta_{12}$.

Proof. Straightforward.

Definition 4.3 A property $P$ of a sbts $\left(X, \eta_{1}, \eta_{2}, E\right)$ is called hereditary property if every soft bitopological subspace $\left(Y, \eta_{1 Y}, \eta_{2 Y}, E\right)$ of $\left(X, \eta_{1}, \eta_{2}, E\right)$ is also has the property $P$.

Remark 4.3 The pairwise soft connectedness does not hereditary property as shown by the following example.

Example 4.5 Let $X=\{x, y, z\}, E=\left\{e_{1}, e_{2}, e_{3}\right\}$ and let 
$\eta_{1}=\{(\tilde{\phi}, E),(\tilde{X}, E),(G, E)\}$,

$\eta_{2}=\{(\tilde{\phi}, E),(\tilde{X}, E),(H, E)\}$

Where,

$(G, E)=\left\{\left(e_{1},\{x\}\right),\left(e_{2},\{x\}\right),\left(e_{3},\{x\}\right)\right\}$,

$(H, E)=\left\{\left(e_{1},\{z\}\right),\left(e_{2},\{z\}\right),\left(e_{3},\{z\}\right)\right\}$.

Then, $\left(X, \eta_{1}, \eta_{2}, E\right)$ is a sbts. Moreover,

$\eta_{12}=\{(\tilde{\phi}, E),(\tilde{X}, E),(G, E),(H, E),(P, E)\}$

Where,

$(P, E)=\left\{\left(e_{1},\{x, z\}\right),\left(e_{2},\{x, z\}\right),\left(e_{3},\{x, z\}\right)\right\}$.

Since $(\tilde{X}, E)$ has no proper soft subset which is both $p$ open and $p$-closed soft set other than $(\tilde{\phi}, E)$, then $\left(X, \eta_{1}, \eta_{2}, E\right)$ is a $p$-soft connected space.

Now, let $Y=\{x, z\}$. It is easily to seen that $(\tilde{Y}, E)=(P, E)$ and so

$\eta_{1 Y}=\{(\tilde{\phi}, E),(\tilde{Y}, E),(G, E)\}$,

$\eta_{2 Y}=\{(\tilde{\phi}, E),(\tilde{Y}, E),(H, E)\}$.

Then,

$\left(Y, \eta_{1 Y}, \eta_{2 Y}, E\right)$ is a soft bitopological subspace of $\left(X, \eta_{1}, \eta_{2}, E\right)$. Furthermore,

$$
\eta_{12 Y}=\{(\tilde{\phi}, E),(\tilde{Y}, E),(G, E),(H, E)\} .
$$

Since $(G, E),(H, E)$ are non-null disjoint $p$-open soft sets in $\left(Y, \eta_{1 Y}, \eta_{2 Y}, E\right) \quad$ and $(\tilde{Y}, E)=(G, E) \tilde{\cup}(H, E)$. Hence, by Theorem 4.2 (3) we deduce that $\left(Y, \eta_{1 Y}, \eta_{2 Y}, E\right)$ is a $p$-soft disconnected space. Therefore, the pairwise soft connectedness does not hereditary property.

Theorem 4.4 Let $\left(X, \sigma_{1}, \sigma_{2}, E\right)$ be a sbts finer than of a sbts $\left(X, \eta_{1}, \eta_{2}, E\right)$. Then,

(1) If $\left(X, \eta_{1}, \eta_{2}, E\right)$ is a $p$-soft disconnected space, then $\left(X, \sigma_{1}, \sigma_{2}, E\right)$ is a $p$-soft disconnected space.

(2) If $\left(X, \sigma_{1}, \sigma_{2}, E\right)$ is a $p$-soft connected space, then $\left(X, \eta_{1}, \eta_{2}, E\right)$ is a $p$-soft connected space.

Proof. (1) : Since $\left(X, \eta_{1}, \eta_{2}, E\right)$ is a $p$-soft disconnected space, then there exist $(G, E),(H, E) \in S S(X)_{E}$ such that $s c l_{\eta_{12}}(G, E) \tilde{\cap}(H, E)=(\tilde{\phi}, E)$

$s c l_{\eta_{12}}(H, E) \tilde{\cap}(G, E)=(\tilde{\phi}, E)$ and

$(G, E) \tilde{\cup}(H, E)=(\tilde{X}, E)$. Now, since

$\left(X, \sigma_{1}, \sigma_{2}, E\right)$ is a finer than of $\left(X, \eta_{1}, \eta_{2}, E\right)$, then

$\eta_{12} \subseteq \sigma_{12}$. It follows that for any soft set $(G, E)$ we have $s c l_{\sigma_{12}}(G, E) \widetilde{\subseteq} s c l_{\eta_{12}}(G, E)$ [by Theorem

2.5].Consequently, $\operatorname{scl}_{\sigma_{12}}(G, E) \tilde{\cap}(H, E)=(\tilde{\phi}, E)$, $s c l_{\sigma_{12}}(H, E) \tilde{\cap}(G, E)=(\tilde{\phi}, E)$ and $(G, E) \tilde{\cup}(H, E)=(\tilde{X}, E)$. Hence, $\left(X, \sigma_{1}, \sigma_{2}, E\right)$ is a $p$-soft disconnected space.

(2): Suppose that $\left(X, \sigma_{1}, \sigma_{2}, E\right)$ is a $p$-soft connected space. Assume that $\left(X, \eta_{1}, \eta_{2}, E\right)$ is a $p$-soft disconnected space. Then, by $(1),\left(X, \sigma_{1}, \sigma_{2}, E\right)$ is a $p$-soft disconnected space, a contradiction.

Theorem 4.5 Let $\left(X, \eta_{1}, \eta_{2}, E\right)$ be a sbts. Then,

If $\left(X, \eta_{1}, E\right)$ or $\left(X, \eta_{2}, E\right)$ is a soft disconnected space, then $\left(X, \eta_{1}, \eta_{2}, E\right)$ is a $p$-soft disconnected space.

Proof. It is immediate from the fact that $s c l_{\eta_{12}}(G, E)=s c l_{\eta_{1}}(G, E) \tilde{\cap} s c l_{\eta_{2}}(G, E)$

[Corollary 3.15 in [13]].

Remark 4.4 Let $\left(X, \eta_{1}, \eta_{2}, E\right)$ be a sbts. If $\left(X, \eta_{1}, E\right)$ and $\left(X, \eta_{2}, E\right)$ are both soft connected spaces, then $\left(X, \eta_{1}, \eta_{2}, E\right)$ may not be a $p$-soft connected space as shown by the following example.

Example 4.6 Let $X=\{a, b\}, E=\left\{e_{1}, e_{2}\right\}$ and let

$$
\eta_{1}=\{(\tilde{\phi}, E),(\tilde{X}, E),(M, E)\},
$$

$\eta_{2}=\{(\tilde{\phi}, E),(\tilde{X}, E),(N, E)\}$

where,

$(M, E)=\left\{\left(e_{1},\{a\}\right),\left(e_{2},\{b\}\right)\right\}$,

$(N, E)=\left\{\left(e_{1},\{b\}\right),\left(e_{2},\{a\}\right)\right\}$. Then, $\left(X, \eta_{1}, E\right)$ and $\left(X, \eta_{2}, E\right)$ are soft connected spaces. Obvious that $\left(X, \eta_{1}, \eta_{2}, E\right)$ is a sbts. Moreover,

$$
\eta_{12}=\{(\tilde{\phi}, E),(\tilde{X}, E),(M, E),(N, E)\} \text {. Since }
$$
$(M, E),(N, E)$ are non-null disjoint $p$-open soft sets and $(\tilde{X}, E)=(M, E) \tilde{\cup}(N, E)$. Hence, by Theorem 4.2 (3) we deduce that $\left(X, \eta_{1}, \eta_{2}, E\right)$ is a $p$-soft disconnected space. 
Theorem 4.6 Let $\left(X, \eta_{1}, \eta_{2}, E\right)$ be a sbts, $Y \subseteq X$ and let $\left(Y, \eta_{1 Y}, \eta_{2 Y}, E\right)$ be a $p$-soft connected space. If $(G, E)$ and $(H, E)$ are $p$-soft separation of $(\tilde{X}, E)$, then $(\tilde{Y}, E) \widetilde{\subseteq}(G, E)$ or $(\tilde{Y}, E) \widetilde{\subseteq}(H, E)$.

Proof. Assume that $(\tilde{Y}, E) \tilde{U}(G, E) \quad$ and $(\tilde{Y}, E) \tilde{U}(H, E)$. Since $(G, E)$ and $(H, E)$ are $p$ soft separation of $(\tilde{X}, E), \quad$ then $(\tilde{Y}, E) \widetilde{\simeq}(\tilde{X}, E)=(G, E) \widetilde{\cup}(H, E) \quad$ implies $(\tilde{Y}, E) \tilde{\cap}[(G, E) \widetilde{\cup}(H, E)]=(\tilde{Y}, E)$. It follows that $[(\tilde{Y}, E) \tilde{\cap}(G, E)] \tilde{\cup}[(\tilde{Y}, E) \tilde{\cap}(H, E)]=(\tilde{Y}, E)$.

On the other hand, since $(\tilde{Y}, E) \tilde{U}(G, E)$, $(\tilde{Y}, E) \tilde{U}(H, E)$ and $(\tilde{Y}, E) \widetilde{\subseteq}(G, E) \tilde{\cup}(H, E)$, then $\quad(\tilde{\phi}, E) \neq(\tilde{Y}, E) \tilde{\cap}(G, E) \neq(\tilde{Y}, E)$, $(\tilde{\phi}, E) \neq(\tilde{Y}, E) \tilde{\cap}(H, E) \neq(\tilde{Y}, E)$. Now, since $(G, E) \tilde{\cap}(H, E)=(\tilde{\phi}, E)$ and

$$
s c l_{\eta_{12 Y}}(G, E) \widetilde{\subseteq}(\tilde{Y}, E) \tilde{\cap} s c l_{\eta_{12}}(G, E),
$$

then

$s c l_{\eta_{12 Y}}[(\tilde{Y}, E) \tilde{\cap}(G, E)] \tilde{\cap}[(\tilde{Y}, E) \tilde{\cap}(H, E)]=(\tilde{\phi}, E)$ and

$s c l_{\eta_{12 Y}}[(\tilde{Y}, E) \tilde{\cap}(H, E)] \tilde{\cap}[(\tilde{Y}, E) \tilde{\cap}(G, E)]=(\tilde{\phi}, E)$ . Therefore, $(\tilde{Y}, E) \tilde{\cap}(G, E),(\tilde{Y}, E) \tilde{\cap}(H, E)$ are $p$-soft separation of $(\tilde{Y}, E)$ which contradicts with that $\left(Y, \eta_{1 Y}, \eta_{2 Y}, E\right)$ is a $p$-soft connected space. Hence, our assumption is not true. Thus, $(\tilde{Y}, E) \widetilde{\subseteq}(G, E)$ or $(\tilde{Y}, E) \simeq(H, E)$.

Theorem 4.7 Let $\left(X, \eta_{1}, \eta_{2}, E\right)$ be a sbts. If $\left(X, \eta_{1}, \eta_{2}, E\right)$ is a $p$-soft disconnected, then $\left(X, \eta_{p \Lambda}, E\right)$ is a soft disconnected.

Proof. Straightforward.

Theorem 4.8 Let $\left(X, \eta_{1}, \eta_{2}, E\right)$ be a sbts. If $\left(X, \eta_{p \Lambda}, E\right)$ is a soft connected, then $\left(X, \eta_{1}, \eta_{2}, E\right)$ is a $p$-soft connected.

Proof. Straightforward.

\section{PAIRWISE SOFT CONNECTED (DISCONNECTED) SOFT SETS}

Definition 5.1 A soft set $(G, E)$ in a sbts $\left(X, \eta_{1}, \eta_{2}, E\right)$ is said to be a pairwise disconnected soft set [briefly, $p$ disconnected soft set] if there exist two non-null p-open soft sets $\left(O_{1}, E\right),\left(O_{2}, E\right)$ such that $(G, E) \tilde{\cap}\left(O_{1}, E\right) \neq(\tilde{\phi}, E)$, $(G, E) \tilde{\cap}\left(O_{2}, E\right) \neq(\tilde{\phi}, E)$, $(G, E) \widetilde{\simeq}\left(O_{1}, E\right) \widetilde{\sim}\left(O_{2}, E\right)$ and $\left(O_{1}, E\right) \widetilde{\cap}\left(O_{2}, E\right) \widetilde{\subseteq}(G, E)^{c}$. In this case we say that $\left(O_{1}, E\right) \sim\left(O_{2}, E\right)$ are $p$-soft disconnection of $(G, E)$ . A soft set $(G, E)$ is called a $p$-connected soft set if has no $p$-soft disconnection.

Example 5.1 From Example 3.1, let $(F, E)=\left\{\left(e_{1},\{x, y\}\right),\left(e_{2},\{y, w\}\right)\right\}$. Take $\left(O_{1}, E\right)=(G, E),\left(O_{2}, E\right)=(H, E)$. It is clear that $(F, E) \tilde{\cap}\left(O_{1}, E\right) \neq(\tilde{\phi}, E)$,

$(F, E) \tilde{\cap}\left(O_{2}, E\right) \neq(\tilde{\phi}, E)$,

$(F, E) \simeq\left(O_{1}, E\right) \widetilde{\cup}\left(O_{2}, E\right)=(\tilde{X}, E)$ and $\left(O_{1}, E\right) \tilde{\cap}\left(O_{2}, E\right)=(\tilde{\phi}, E) \widetilde{\subseteq}(G, E)^{c}$. Hence, $(F, E)$ is a $p$-disconnected soft set.

Theorem 5.1 Every $p$-connected soft set in a sbts

$\left(X, \eta_{1}, \eta_{2}, E\right)$ is a connected soft set in sbts $\left(X, \eta_{p \Lambda}, E\right)$,

Proof. Straightforward.

Lemma 5.1 If $\left(O_{1}, E\right) \widetilde{\cup}\left(O_{2}, E\right)$ are $p$-soft disconnection of $(G, E)$ in a sbts $\left(X, \eta_{1}, \eta_{2}, E\right)$, then $(G, E) \tilde{\cap}\left(O_{1}, E\right)$ and $(G, E) \tilde{\cap}\left(O_{2}, E\right)$ are $p$ separated soft sets.

Proof. Since $\left(O_{1}, E\right) \widetilde{\cup}\left(O_{2}, E\right)$ is a $p$-soft disconnection of $(G, E)$, then

$(G, E) \tilde{\cap}\left(O_{1}, E\right) \neq(\tilde{\phi}, E)$,

$(G, E) \tilde{\cap}\left(O_{2}, E\right) \neq(\tilde{\phi}, E)$,

$(G, E) \simeq\left(O_{1}, E\right) \widetilde{\cup}\left(O_{2}, E\right)$ and

$\left(O_{1}, E\right) \tilde{\cap}\left(O_{2}, E\right) \widetilde{\subseteq}(G, E)^{c}$. We shall prove that $\left(O_{1}, E\right) \tilde{\cap}(G, E),\left(O_{2}, E\right) \tilde{\cap}(G, E)$ are $p$-separated soft sets.

Let $x_{e} \tilde{\in} \operatorname{scl}_{12}\left[\left(O_{1}, E\right) \tilde{\cap}(G, E)\right]$. Then, by Theorem $2.5\left[\left(O_{1}, E\right) \tilde{\cap}(G, E)\right] \tilde{\cap}\left(O_{x_{e}}, E\right) \neq(\tilde{\phi}, E)$, $\forall\left(O_{x_{e}}, E\right) \in \eta_{12}\left(x_{e}\right)$. Now, assume that $x_{e} \tilde{\in}\left[\left(O_{2}, E\right) \tilde{\cap}(G, E)\right]$. It follows that 
$x_{e} \tilde{\in}\left(O_{2}, E\right)$. Therefore, $\left(O_{2}, E\right) \in \eta_{12}\left(x_{e}\right)$. Thus, $\left[\left(O_{1}, E\right) \tilde{\cap}(G, E)\right] \tilde{\cap}\left(O_{2}, E\right) \neq(\tilde{\phi}, E) \quad$ which $\quad$ a contradicts with the given $\left(O_{1}, E\right) \tilde{\cap}\left(O_{2}, E\right) \tilde{\subseteq}(G, E)^{c}$.

Hence,

$x_{e} \notin\left[\left(O_{2}, E\right) \tilde{\cap}(G, E)\right]$. Consequently, $\operatorname{scl}_{12}\left[\left(O_{1}, E\right) \tilde{\cap}(G, E)\right] \tilde{\cap}\left[\left(O_{2}, E\right) \tilde{\cap}(G, E)\right]=(\tilde{\phi}, E)$ Similarly, $\operatorname{scl}_{12}\left[\left(O_{2}, E\right) \tilde{\cap}(G, E)\right] \tilde{\cap}\left[\left(O_{1}, E\right) \tilde{\cap}(G, E)\right]=(\tilde{\phi}, E)$ . Hence, $\left(O_{1}, E\right) \tilde{\cap}(G, E),\left(O_{2}, E\right) \tilde{\cap}(G, E)$ are $p$ separated soft sets.

Theorem 5.2 A soft set $(G, E)$ in a sbts $\left(X, \eta_{1}, \eta_{2}, E\right)$ is a $p$-disconnected soft set iff there exist two $p$-separated soft sets $\left(S_{1}, E\right),\left(S_{2}, E\right)$ such that $(G, E)=\left(S_{1}, E\right) \widetilde{\cup}\left(S_{2}, E\right)$

Proof. $\Rightarrow$ : Suppose that $(G, E)$ is a $p$ disconnected soft set in $\left(X, \eta_{1}, \eta_{2}, E\right)$. Then $(G, E)$ has a $p$-soft disconnection, say $\left(O_{1}, E\right) \widetilde{\cup}\left(O_{2}, E\right)$, i.e., there exist two non-null $p$-open soft sets $\left(O_{1}, E\right),\left(O_{2}, E\right)$ such that

$(G, E) \tilde{\cap}\left(O_{1}, E\right) \neq(\tilde{\phi}, E)$,

$(G, E) \tilde{\cap}\left(O_{2}, E\right) \neq(\tilde{\phi}, E)$,

$(G, E) \simeq\left(O_{1}, E\right) \widetilde{\cup}\left(O_{2}, E\right)$

and

$\left(O_{1}, E\right) \tilde{\cap}\left(O_{2}, E\right) \widetilde{\subseteq}(G, E)^{c}$. It follows by Lemma 5.1 that $(G, E) \tilde{\cap}\left(O_{1}, E\right)$ and $(G, E) \tilde{\cap}\left(O_{2}, E\right)$ are $p$ separated soft sets. Since $(G, E) \simeq\left(O_{1}, E\right) \widetilde{\cup}\left(O_{2}, E\right)$, then

implies

$$
(G, E) \tilde{\cap}\left[\left(O_{1}, E\right) \sim\left(O_{2}, E\right)\right]=(G, E)
$$

$\left[(G, E) \tilde{\cap}\left(O_{1}, E\right)\right] \tilde{\cup}\left[(G, E) \tilde{\cap}\left(O_{2}, E\right)\right]=(G, E)$.

Take $\quad\left(S_{1}, E\right)=\left(O_{1}, E\right) \tilde{\cap}(G, E) \quad$ and

$\left(S_{2}, E\right)=\left(O_{2}, E\right) \tilde{\cap}(G, E)$.

$\Leftarrow$ : Let $\left(S_{1}, E\right),\left(S_{2}, E\right)$ be two $p$-separated soft sets and let $(G, E) \in S S(X)_{E}$ such that

$(G, E)=\left(S_{1}, E\right) \sim\left(S_{2}, E\right)$. Then

$\operatorname{scl}_{12}\left(S_{1}, E\right) \tilde{\cap}\left(S_{2}, E\right)=(\tilde{\phi}, E)$ and

$\operatorname{scl}_{12}\left(S_{2}, E\right) \tilde{\cap}\left(S_{1}, E\right)=(\tilde{\phi}, E)$. Take

$\left(O_{1}, E\right)=\left[\operatorname{scl}_{12}\left(S_{1}, E\right)\right]^{c}$ and

$\left(O_{2}, E\right)=\left[s c l_{12}\left(S_{2}, E\right)\right]^{c}$. So, $\left(O_{1}, E\right),\left(O_{2}, E\right)$ are non-null $p$-open soft sets. Since

$\operatorname{scl}_{12}\left(S_{2}, E\right) \tilde{\cap}\left(S_{1}, E\right)=(\tilde{\phi}, E)$, then
$\left(S_{1}, E\right) \widetilde{\widetilde{C}}\left[s c l_{12}\left(S_{2}, E\right)\right]^{c}=\left(O_{2}, E\right)$. By similar we also have $\left(S_{2}, E\right) \simeq\left(O_{1}, E\right)$. It follows that $(G, E) \simeq\left(O_{1}, E\right) \sim\left(O_{2}, E\right)$. Now, since $\left[\operatorname{scl}_{12}\left(S_{1}, E\right)\right]^{c} \simeq\left(S_{1}, E\right)^{c}$, $\left[\operatorname{scl}_{12}\left(S_{2}, E\right)\right]^{c} \simeq\left(S_{2}, E\right)^{c}$, then $\left(O_{1}, E\right) \tilde{\cap}\left(O_{2}, E\right) \widetilde{\subseteq}(G, E)^{c}$. Furthermore, since $\left(S_{1}, E\right),\left(S_{2}, E\right) \simeq(G, E)$ and $\left(S_{2}, E\right) \simeq\left(O_{1}, E\right)$, $\left(S_{1}, E\right) \simeq\left(O_{2}, E\right)$, then

$\left(S_{2}, E\right) \widetilde{\simeq}(G, E) \widetilde{\cap}\left(O_{1}, E\right)$ and $\left(S_{1}, E\right) \widetilde{\subseteq}(G, E) \widetilde{\cap}\left(O_{2}, E\right)$. But, $\left(S_{1}, E\right) \neq(\tilde{\phi}, E)$, $\left(S_{2}, E\right) \neq(\tilde{\phi}, E)$, then $(G, E) \tilde{\cap}\left(O_{2}, E\right) \neq(\tilde{\phi}, E)$ and $(G, E) \tilde{\cap}\left(O_{1}, E\right) \neq(\tilde{\phi}, E)$. Consequently, $(G, E)$ is a $p$-disconnected soft set.

Corollary 5.1 Let $\left(X, \eta_{1}, \eta_{2}, E\right)$ be a sbts. If $\left(S_{1}, E\right),\left(S_{2}, E\right)$ are two $p$-separated soft sets, then $\left(S_{1}, E\right) \widetilde{\cup}\left(S_{2}, E\right)$ is a $p$-disconnected soft set.

Corollary 5.2 A soft set $(G, E)$ in a sbts $\left(X, \eta_{1}, \eta_{2}, E\right)$ is said to be a $p$-connected soft set iff cannot expressed as a union of two $p$-separated soft sets.

Proposition 5.1 Let $\left(X, \eta_{1}, \eta_{2}, E\right)$ be a sbts. Then

(1) Every soft point is $p$-connected soft set.

(2) The null soft set is $p$-connected soft set.

Proof. (1): Let $\left(x_{e}, E\right)$ be a soft point in $(\tilde{X}, E)$ Then, for any two non-null $p$-open soft sets $\left(O_{1}, E\right),\left(O_{2}, E\right)$ such that $\left(x_{e}, E\right) \tilde{\cap}\left(O_{1}, E\right) \neq(\tilde{\phi}, E)$, $\left(x_{e}, E\right) \tilde{\cap}\left(O_{2}, E\right) \neq(\tilde{\phi}, E), \quad$ we have $\left(x_{e}, E\right) \tilde{\epsilon}\left(O_{1}, E\right) \tilde{\cap}\left(O_{2}, E\right)$. It follows that $\left(O_{1}, E\right) \tilde{\cap}\left(O_{2}, E\right)$ Ú $\left(x_{e}, E\right)^{c}$. Hence, $\left(x_{e}, E\right)$ is a $p$. connected soft set.

(2): Obvious.

Theorem 5.3 Let $(F, E)$ be a $p$-connected soft set in a sbts $\left(X, \eta_{1}, \eta_{2}, E\right)$ and let

$$
(F, E) \widetilde{\subseteq}(M, E) \widetilde{\subseteq} s c l_{\eta_{12}}(F, E) . \text { Then }
$$

$(M, E), s c l_{\eta_{12}}(F, E)$ are also $p$-connected soft sets. 
Proof. Let $(F, E)$ be a $p$-connected soft set in a sbts $\left(X, \eta_{1}, \eta_{2}, E\right)$ and assume that $(M, E)$ is a $p$ disconnected soft set in $\left(X, \eta_{1}, \eta_{2}, E\right)$. Then, there exist two non-null $p$-open soft sets $\left(O_{1}, E\right),\left(O_{2}, E\right)$ such that $(M, E) \tilde{\cap}\left(O_{1}, E\right) \neq(\tilde{\phi}, E)$,

$(M, E) \tilde{\cap}\left(O_{2}, E\right) \neq(\tilde{\phi}, E)$,

$(M, E) \widetilde{\subseteq}\left(O_{1}, E\right) \sim\left(O_{2}, E\right)$ and

$\left(O_{1}, E\right) \tilde{\cap}\left(O_{2}, E\right) \widetilde{\simeq}(M, E)^{c}$. Since

$(F, E) \simeq(M, E)$, then

$(F, E) \widetilde{\simeq}\left(O_{1}, E\right) \widetilde{\cup}\left(O_{2}, E\right)$ and

$\left(O_{1}, E\right) \tilde{\cap}\left(O_{2}, E\right) \tilde{\subseteq}(F, E)^{c}$. But $(F, E)$ is a $p$. connected soft set, then either

$(F, E) \tilde{\cap}\left(O_{1}, E\right)=(\tilde{\phi}, E)$ or

$(F, E) \tilde{\cap}\left(O_{2}, E\right)=(\tilde{\phi}, E)$. If we claim that

$(F, E) \tilde{\cap}\left(O_{1}, E\right)=(\tilde{\phi}, E)$, then $\left(O_{1}, E\right)^{c}$ is a $p$ -

closed soft set contains $(F, E)$. It follows that

$s c l_{\eta_{12}}(F, E) \simeq\left(O_{1}, E\right)^{c}$ which implies that

$(M, E) \tilde{\cap}\left(O_{1}, E\right)=(\tilde{\phi}, E)$, a contradicts with our assumption. Hence, our assumption is false. Consequently, $(M, E)$ is a $p$-connected soft set. In particular, put $(M, E)=s c l_{\eta_{12}}(F, E)$, then $s c l_{\eta_{12}}(F, E)$ is also $p$-connected soft set.

Remark 5.1 The soft subset of a $p$-soft connected space need not be a $p$-connected soft set as seen in the following example.

Example 5.2 Consider the $p$-soft connected space

$\left(X, \eta_{1}, \eta_{2}, E\right)$ in Example 4.2. Let

$(F, E)=\left\{\left(e_{1}, \phi\right),\left(e_{2},\{a\}\right),\left(e_{3},\{a\}\right)\right\}$. Take

$\left(O_{1}, E\right)=\left(G_{2}, E\right)$ and $\left(O_{2}, E\right)=\left(H_{2}, E\right)$.

Therefore,

$(F, E) \tilde{\cap}\left(O_{1}, E\right)=\left\{\left(e_{1}, \phi\right),\left(e_{1},\{a\}\right),\left(e_{1}, \phi\right)\right\} \neq(\tilde{\phi}, E)$

$(F, E) \tilde{\cap}\left(O_{2}, E\right)=\left\{\left(e_{1}, \phi\right),\left(e_{1}, \phi\right),\left(e_{1},\{a\}\right)\right\} \neq(\tilde{\phi}, E)$

$(F, E) \widetilde{\subseteq}\left(O_{1}, E\right) \widetilde{\cup}\left(O_{2}, E\right)=\left\{\left(e_{1},\{b\}\right),\left(e_{1},\{a, c\}\right)\right.$

,$\left.\left(e_{1},\{a, d\}\right)\right\}$ and

$\left(O_{1}, E\right) \tilde{\cap}\left(O_{2}, E\right)=\left\{\left(e_{1}, \phi\right),\left(e_{1},\{c\}\right),\left(e_{1},\{d\}\right)\right\} \widetilde{\subseteq}$

$(F, E)^{c}$. Hence, $(F, E)$ is a $p$-disconnected soft subset of $\left(X, \eta_{1}, \eta_{2}, E\right)$.
Remark 5.2 The union of two $p$-connected soft sets need not be a $p$-connected soft set as seen in the following example.

Example 5.3 In Example 5.2, it is clear by Proposition 5.1 (1) that $\left(a_{e_{2}}, E\right),\left(a_{e_{3}}, E\right)$ are $p$-connected soft sets. Nevertheless,

$\left(a_{e_{2}}, E\right) \widetilde{\cup}\left(a_{e_{3}}, E\right)=\left\{\left(e_{1}, \phi\right),\left(e_{2},\{a\}\right),\left(e_{3},\{a\}\right)\right\}=(F, E)$ is a $p$-disconnected soft set.

Theorem 5.4 Let $(G, E),(H, E)$ be two $p$-connected soft sets in sbts $\left(X, \eta_{1}, \eta_{2}, E\right)$. If

$(G, E) \tilde{\cap}(H, E) \neq(\tilde{\phi}, E)$, then $(G, E) \tilde{\cup}(H, E)$ is a $p$-connected soft set.

Proof. Suppose that $(G, E),(H, E)$ are $p$-connected soft sets and $(G, E) \tilde{\cap}(H, E) \neq(\tilde{\phi}, E)$. Assume that $(G, E) \widetilde{\cup}(H, E)$ is a $p$-disconnected soft set. Then there exist two non-null $p$-open soft sets

$\left(O_{1}, E\right),\left(O_{2}, E\right)$ such that

$[(G, E) \widetilde{\cup}(H, E)] \tilde{\cap}\left(O_{1}, E\right) \neq(\tilde{\phi}, E)$,

$[(G, E) \widetilde{\cup}(H, E)] \tilde{\cap}\left(O_{2}, E\right) \neq(\tilde{\phi}, E)$,

$[(G, E) \widetilde{\cup}(H, E)] \widetilde{\subseteq}\left(O_{1}, E\right) \widetilde{\cup}\left(O_{2}, E\right)$ and

$\left(O_{1}, E\right) \tilde{\cap}\left(O_{2}, E\right) \widetilde{\simeq}[(G, E) \widetilde{\cup}(H, E)]^{c}$. Since

$(G, E) \widetilde{\simeq}(G, E) \widetilde{\cup}(H, E)$, then

$(G, E) \widetilde{\subseteq}\left(O_{1}, E\right) \widetilde{\cup}\left(O_{2}, E\right)$ and

$\left(O_{1}, E\right) \tilde{\cap}\left(O_{2}, E\right) \widetilde{\subseteq}(G, E)^{c}$. But $(G, E)$ is a $p$. connected soft set, then $(G, E) \tilde{\cap}\left(O_{1}, E\right)=(\tilde{\phi}, E)$ or $(G, E) \tilde{\cap}\left(O_{2}, E\right)=(\tilde{\phi}, E)$. Therefore,

$(G, E) \simeq\left(O_{1}, E\right)$ or $(G, E) \widetilde{\subseteq}\left(O_{2}, E\right)$ [for $\left.(G, E) \simeq\left(O_{1}, E\right) \sim\left(O_{2}, E\right)\right]$. Similarly,

$(H, E) \simeq\left(O_{1}, E\right)$ or $(H, E) \simeq\left(O_{2}, E\right)$. Thus, if

$(G, E) \simeq\left(O_{1}, E\right)$ and $(H, E) \simeq\left(O_{2}, E\right)$, then

$(G, E) \tilde{\cap}(H, E) \widetilde{\subseteq}\left(O_{1}, E\right) \tilde{\cap}\left(O_{2}, E\right) \widetilde{\subseteq}(G, E)^{c} \tilde{\cap}(H, E)^{c}$

which implies that $(G, E) \tilde{\cap}(H, E)=(\tilde{\phi}, E)$, a

contradiction. Similarly when $(G, E) \simeq\left(O_{2}, E\right)$ and

$(H, E) \widetilde{\subseteq}\left(O_{1}, E\right)$ we have a contradiction.

Consequently, our assumption is not true. Hence,

$(G, E) \widetilde{\cup}(H, E)$ is a $p$-connected soft set.

Theorem 5.5 Let $f_{p u}$ be a $p$-soft continuous mapping from a sbts $\left(X, \eta_{1}, \eta_{2}, E\right)$ into a sbts $\left(Y, \sigma_{1}, \sigma_{2}, K\right)$. If

$(G, E)$ is a $p$-connected soft set in $\left(X, \eta_{1}, \eta_{2}, E\right)$, 
then $f_{p u}(G, E)$ is a $p$-connected soft set in

$\left(Y, \sigma_{1}, \sigma_{2}, K\right)$.

Proof. Let $(G, E)$ be a $p$-connected soft set in

$\left(X, \eta_{1}, \eta_{2}, E\right)$. Assume that $f_{p u}(G, E)$ is not $p$ -

connected soft set in $\left(Y, \sigma_{1}, \sigma_{2}, K\right)$. Then there exist two

non-null $p$-open soft sets $\left(O_{1}, K\right),\left(O_{2}, K\right)$ in

$\left(Y, \sigma_{1}, \sigma_{2}, K\right)$ such that

$f_{p u}(G, E) \tilde{\cap}\left(O_{1}, K\right) \neq(\tilde{\phi}, K)$,

$f_{p u}(G, E) \tilde{\cap}\left(O_{2}, K\right) \neq(\tilde{\phi}, K)$,

$f_{p u}(G, E) \simeq\left(O_{1}, K\right) \widetilde{\sim}\left(O_{2}, K\right)$ and

$\left(O_{1}, K\right) \tilde{\cap}\left(O_{2}, K\right) \widetilde{\subseteq}\left[(\tilde{Y}, K) \backslash f_{p u}(G, E)\right]$. It

follows by Theorems 2.2 and 2.3 that

$(G, E) \tilde{\cap} f_{p u}^{-1}\left(O_{1}, K\right) \neq(\tilde{\phi}, E)$,

$(G, E) \tilde{\cap} f_{p u}^{-1}\left(O_{2}, K\right) \neq(\tilde{\phi}, E)$,

$(G, E) \widetilde{\simeq} f_{p u}^{-1}\left(O_{1}, K\right) \widetilde{\cup} f_{p u}^{-1}\left(O_{2}, K\right)$ and

$f_{p u}^{-1}\left(O_{1}, K\right) \tilde{\cap} f_{p u}^{-1}\left(O_{2}, K\right) \widetilde{\subseteq} f_{p u}^{-1}\left[(\tilde{Y}, K) \backslash f_{p u}(G, E)\right]$

$=(\tilde{X}, E) \backslash(G, E)$. Since $f_{p u}$ is a $p$-soft continuous,

then $f_{p u}^{-1}\left(O_{1}, K\right), f_{p u}^{-1}\left(O_{2}, K\right)$ are $p$-open soft sets in

$\left(X, \eta_{1}, \eta_{2}, E\right)$. Hence, $f_{p u}^{-1}\left(O_{1}, K\right) \tilde{\cup} f_{p u}^{-1}\left(O_{2}, K\right)$

form a $p$-soft disconnection of $(G, E)$ which contrary to

the fact that $(G, E)$ is a $p$-connected soft set in

$\left(X, \eta_{1}, \eta_{2}, E\right)$. Hence, $f_{p u}(G, E)$ is a $p$-connected

soft set in $\left(Y, \sigma_{1}, \sigma_{2}, K\right)$.

Corollary 5.3 Let $f_{p u}$ be a $p$-soft continuous mapping

from a $p$-soft connected space $\left(X, \eta_{1}, \eta_{2}, E\right)$ onto a sbts

$\left(Y, \sigma_{1}, \sigma_{2}, K\right)$. Then $\left(Y, \sigma_{1}, \sigma_{2}, K\right)$ is a $p$-soft

connected space.

\section{CONCLUSION}

Soft bitopological spaces based on soft set which is a collection of information granules is the mathematical formulation of approximate reasoning about information systems. In this paper, we introduced and studied the notion of pairwise separated soft. Based on this notion, we defined and studied some properties and characterizations of pairwise soft connected spaces and pairwise connected soft sets in soft bitopological spaces. Some properties of such notions are obtained. We expect that the _findings in this paper can be promoted to the further study on soft bitopology to carry out general framework for the practical life applications.

\section{ACKNOWLEDGEMENTS}

The authors express their sincere thanks to the reviewers for their valuable suggestions. The authors are also thankful to the editors-in-chief and managing editors for their important comments which helped to improve the presentation of the paper

\section{REFERENCES}

[1] M. Al-Khafaj and M. Mahmood, Some properties of soft connected spaces and soft locally connected spaces, IOSR Journal of Mathematics 10 (5) (2014), 102 - 107.

[2] A. Aygunoglu and H. Aygun, Some notes on soft topological spaces, Neural Comput. Applic. 21 (Suppl 1) (2012) S113-S119.

[3] S. Das and S. K. Samanta, Soft metric, Ann. Fuzzy Math. Inform. 6 (2013) $77-94$.

[4] S. A. El-Sheikh, Dimension Theory of Bitopological Spaces, Master Thesis, Ain Shams University, Cairo, Egypt, 1987.

[5] S. A. El-Sheikh and A. M. Abd El-latif, Decompositions of some types of supra soft sets and soft continuity, International Journal of Mathematics Trends and Technology 9 (1) (2014) $37-56$.

[6] D. N. Georgiou a and A. C. Megaritis, Soft set theory and topology, Appl. Gen. Topol. 15 (1) (2014) 93- 109.

[7] H. Hazra, P. Majumdar and S. K.Samanta, Soft topology, Fuzzy Inform. Eng. 4 (1) (2012) $105-115$.

[8] S. Hussain and B. Ahmad, Some properties of soft topological spaces, Comput. Math. Appl. 62 (2011) 4058 -4067 .

[9] M. Irfan Ali, M. Shabir and M. Naz, Algebraic structures of soft sets associated with new operations, Comput. Math. Appl. 61 (2011) 2647-2654.

[10] B. M. Ittanagi, Soft bitopological spaces, International journal of Computer Applications 107 (7) (2011) 1 -4.

[11] A. Kandil, O. A. E. Tantawy, S. A. El-Sheikh and Shawqi A. Hazza, Generalized pairwise closed soft sets and the associate pairwise soft separation axioms, South Asian J. Math. 6 (2) (2016) 43-57.

[12] A. Kandil, O. A. E. Tantawy, S. A. El-Sheikh and Shawqi A. Hazza, On pairwise $\lambda$-open soft sets and pairwise locally closed soft sets, American Scientific Research Journal for Engineering, Technology, and Sciences 28 (1) (2017) 225-247.

[13] A. Kandil, O. A. E. Tantawy, S. A. El-Sheikh and Shawqi A. Hazza, Pairwise open (closed) soft sets in a soft bitopological spaces, Ann. Fuzzy Math. Inform. 11 (4) (2016) $571-588$.

[14] A. Kandil, O. A. E. Tantawy, S. A. El-Sheikh and Shawqi A. Hazza, Pairwise soft separation axioms in soft bitopological spaces, Ann. Fuzzy Math. Inform. 13 (5) (2017) $563-577$.

[15] A. Kandil, O. A. E. Tantawy, S. A. El-Sheikh and Shawqi A. Hazza, Some types of pairwise soft sets and the associated soft topologies, Journal of Intelligent and Fuzzy Syst 32 (2) (2017) 1007 - 1018.

[16] A. Kandil, O. A. E. Tantawy, S. A. El-Sheikh and Shawqi A. Hazza, Some types of pairwise soft open (continuous) mappings and some related results, South Asian J. Math. 7 (2) (2017) 1-21. 
[17] A. Kharal and B. Ahmad, Mappings on soft classes, New Math. Nat. Comput. 7 (3) (2011) 471 - 481.

[18] P. Majumdar and S. K. Samanta, On soft mappings, Comput. Math. Appl. 60 (2010) 2666 - 2672.

[19] D. Molodtsov, Soft set theory-First results, Comput. Math. Appl. 37 (1999) 19-31.

[20] Sk. Nazmul and S. K. Samanta, Neighbourhood properties of soft topological spaces, Ann. Fuzzy Math. Inform. 6 (1) (2013) 1-15.

[21] Sk. Nazmul and S. K. Samanta, Some properties of soft topologies and group soft topologies, Ann. Fuzzy Math. Inform. 8 (4) (2014) 645-661.

[22] Ningxin Xie, Soft points and the structure of soft topological spaces, Ann. Fuzzy. Math. inform. 10 (2) (2015) 309-322.
[23] E. Peyghan, B. Samadi and A. Tayebi, About soft topological spaces, Journal of New Results in Science 2 (2013) 60-75.

[24] E. Peyghan, B. Samadi and A. Tayebi, On soft connectedness arXiv 1202.1668v1,math.GN,(2012).

[25] D. Pie and D. Miao, From soft sets to information systems, Granular computing, IEEE Inter. Conf. 2 (2005) $617-621$.

[26] M. Shabir and M. Naz, On soft toplogical spaces, Comput. Math. Appl. 61 (7) (2011) 1786 - 1799.

[27] I. Zorlutuna, M. Akdag, W. K. Min and S. Atmaca, Remarks on soft topological spaces, Ann. Fuzzy Math. Inform. 3 (2) (2012) $171-185$.

[28] I. Zorlutuna and H. Çakir, On continuity of soft mapping, Appl. Math. Inf. Sci. 1 (9) (2015) 403-409. 